\title{
Quantum-electrodynamical treatment of second-harmonic generation through phase-conjugate six-wave mixing: Temporal analysis
}

\author{
Ian D. Hands, Shujie Lin, Stephen R. Meech, and David L. Andrews* \\ School of Chemical Sciences, University of East Anglia, Norwich NR4 7TJ, United Kingdom
}

(Received 29 September 1999; published 18 July 2000)

\begin{abstract}
It is shown how the effects of molecular reorientation may be incorporated in a fully quantized quantumelectrodynamical treatment of a high-order nonlinear optical effect. Specifically, a general temporal theory is developed to account for the second-harmonic intensity produced through phase-conjugate six-wave mixing. The theory permits elucidation of the intensity of the second-harmonic radiation for arbitrary arrangements of the generating laser beams and molecular geometry. Several models are considered: a one-dimensional model, linear geometry, and a planar geometry. A comparison is made between the results associated with these models and with those obtained from ultrafast experiments on dilute solutions of substituted stilbenes. We find that the off-axial components of the molecular polarizabilities are necessary to properly describe the orientational dynamics of such molecules.

PACS number(s): 42.65.Ky, 78.47.+p, 42.50.Ct, 42.40.Ht
\end{abstract}

\section{INTRODUCTION}

Recently it has been demonstrated that ultrafast highorder nonlinear optical experiments provide novel information that is unavailable through lower-order measurements [1-11]. Current applications include study of the ultrafast dynamics of pure liquids [1-4], orientational dynamics in solution [5-8], and the observation of intermolecular coupling [9]. In addition there are proposals for the use of such measurements in the determination of molecular hyperpolarizabilities [10], and the structure of molecular aggregates [11].

It has also become clear that there is a compelling need for detailed theoretical treatments of higher-order nonlinear optical interaction. High-order experiments produce numerous signals, and their spatial overlap and interferences can render interpretation difficult [12-15]. One method to address the problem of distinguishing between these various signals is to exploit their polarization dependence $[16,17]$. In a previous paper we presented a detailed quantumelectrodynamical treatment of six-wave mixing; the theory was then used to predict and analyze the results of a number of polarization-resolved measurements of the generation in isotropic solutions of optical second harmonics [17]. The purpose of the present paper is to extend that quantumelectrodynamical treatment to the time-resolved regime. Although our primary objective is to provide a complete description of our recent ultrafast time-resolved experiments $[5,6]$, the very general methods developed here also provide a complete framework for application to other time-resolved measurements of six-wave mixing.

This paper is structured as follows. In the following section a brief description of the experiments is given. In Sec. III the temporal theory is described in some detail: first a quantum-electrodynamical analog of the classical transient

\footnotetext{
*Author to whom correspondence should be addressed.
}

grating scattering signal is derived, followed by the introduction of population and orientation dynamics, in the latter case extending the treatment of Favro [18]. In Sec. IV the main predictions of the theory for molecules of different symmetry types are examined and compared with experimental observations. In the final section the conclusions are summarized.

Before proceeding, a brief comment may be made on our choice of a quantum electrodynamical (QED) representation for the theory in Sec. III. With proper caution results of the same form, and leading to precisely the same analysis of orientational diffusion, could be obtained from what is to many the more familiar semiclassical or nonlinear polarization formalism - and for descriptive purposes, that is a language we have used elsewhere [19]. However, for the development of fundamental theory the semiclassical formalism is seriously flawed in a number of respects. For example, the semiclassical expansion of the electric polarization field engenders a sum of quantum amplitudes between processes with nonidentical sets of initial and final radiation states, violating the superposition principle-though in practice, miscreant interference terms are ignored. Also the semiclassical tradition leads to conclusions that disrespect several principles of time-reversal symmetry, for example, in the formal equivalence between the amplitudes for second harmonic generation and degenerate down-conversion [20,21]. QED is the only theory in which the photon concept can be used with legitimacy, and we embrace its rigor.

\section{EXPERIMENTAL DETAILS}

The optical configuration to be considered is shown diagrammatically in Fig. 1, the exact details of which have been reported elsewhere in the literature $[5,16]$. The beams are referred to in terms of the modes to which their photons belong. Photons of mode $1\left(\mathbf{m}_{1}\right)$, are at the laser's fundamental wavelength of $800 \mathrm{~nm}$ and propagate with a wave vector $\mathbf{k}$ and polarization state $\lambda$. This beam is the probe-it may be time-delayed with respect to the seeding beams $\mathbf{m}_{2}$ and $\mathbf{m}_{3}$ by use of computer-controlled optical delays. Beam 


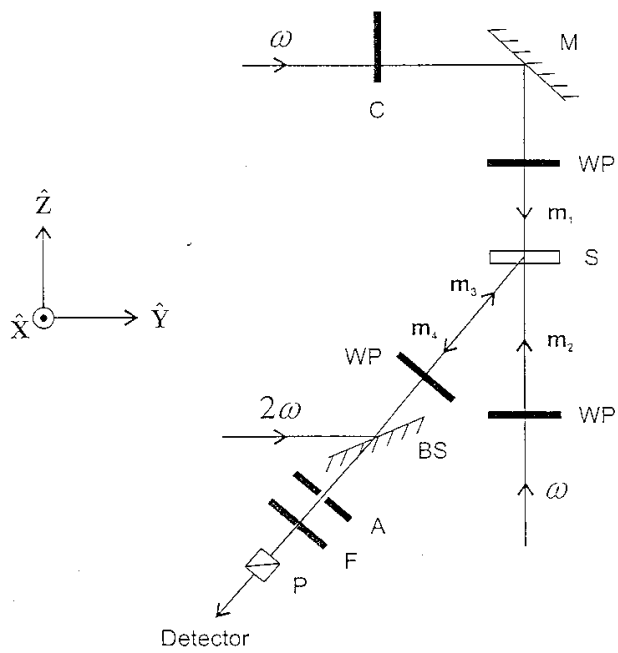

FIG. 1. Schematic diagram of the six-wave mixing arrangement. The fundamental beams counterpropagate with $\mathbf{m}_{1}=(\mathbf{k}, \lambda)$ and $\mathbf{m}_{2}$ $=\left(\mathbf{k}, \lambda^{\prime}\right)$. Mode $\mathbf{m}_{2}$ is a pump beam and arrives at the sample $S$ synchronously with the other (harmonic) pump beam $\mathbf{m}_{3}$ $=\left(\mathbf{k}^{\prime}, \lambda^{\prime \prime}\right)$. Probe beam $\mathbf{m}_{1}$ is time delayed with respect to the pump beams, the harmonic signal $\mathbf{m}_{4}=\left(-\mathbf{k}^{\prime}, \lambda^{\prime \prime \prime}\right)$ being produced in the phase-conjugate direction. Laboratory axes are as shown and other symbols represent A, aperture; BS, beam splitter (50:50 at $400 \mathrm{~nm}$ ); C, chopper; F, 400-nm band-pass filter; P, polarizer; and WP, wave plate.

$\mathbf{m}_{2}$ is also at the fundamental laser wavelength-it propagates in the opposite direction to the probe beam and has an independently adjustable polarization state. Beam $\mathbf{m}_{3}$ is a second harmonic produced on passing some of the laser output through a beta barium borate $(\mathrm{BBO})$ crystal. This second seeding beam makes a small angle $\left(\sim 5^{\circ}\right)$ with beam $\mathbf{m}_{2}$. The second-harmonic signal beam $\mathbf{m}_{4}$ is detected in a direction opposite to $\mathbf{m}_{3}$. The path lengths of the seeding beams are carefully adjusted to ensure that they arrive coincidentally at the sample $S$, which, in our experiments comprise ca. $10^{-3} M$ solutions of either 4-dimethylamino- $4^{\prime}$-nitrostilbene (DMANS) or 4-diethylamino-4'-nitrostilbene (DEANS), in either toluene or tetrahydrofuran. For reference, the structure of DMANS is shown later, in Fig. 6.

\section{THEORY}

To address the dynamical features of second-harmonic emission arising from the experiment described in Sec. II, theory must properly accommodate the designed engagement of optical resonances. The sample is specifically chosen to be absorbing at the harmonic frequency, in order to create the population imbalance responsible for the dynamical behavior (see below). In this regard, weaker signals associated with off-resonance six-wave coherence can only represent a negligible and effectively time-independent background. The coherence timescales over which such signals will exhibit oscillatory features are too short to be significant in the reported experiments, and the secular resonances, which they can enjoin [22] do not lead to population redistribution. Cognizance of the operational conditions thus enables us to focus

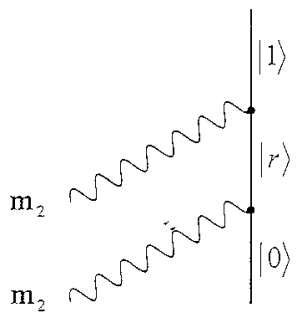

(a)

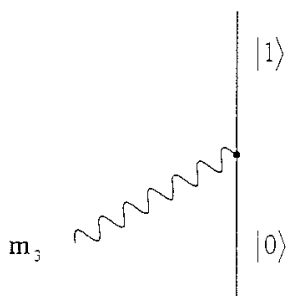

(b)
FIG. 2. Feynman diagrams associated with grating formation: (a) two-photon and (b) single-photon absorption.

on the production of the signal harmonic through the operation of a population grating associated with on-resonance processes, the time dependence of the primary absorption correctly represented by Fermi's golden rule.

\section{A. Grating description}

The first task in this section is to show that, in the presence of the two writing beams $\mathbf{m}_{2}$ and $\mathbf{m}_{3}$, the created population grating is of just the correct periodicity to efficiently generate phase-matched second-harmonic photons from the probe beam $\mathbf{m}_{1}$. As a result, the $\mathbf{m}_{4}$ signal photons emerge at the second-harmonic frequency and propagate in exactly the opposite direction to the seeding beam $\mathbf{m}_{3}$, according to the dictates of wave-vector matching.

We shall suppose that the seeding pulses from modes 2 and 3 are coincident with the sample at time $t=0$ and then at $t=\tau$ the pulse from the probe beam (mode 1 ) arrives. The sample is absorbing at the harmonic frequency and so transition to the excited state is expected. Nonetheless, there are two ways in which this may be accomplished in the presence of the two seeding beams: two-photon absorption of photons solely from the fundamental beam, and single-photon absorption of photons from the harmonic beam. We thus need to consider two Feynman diagrams as shown in Fig. 2. The matrix element (quantum amplitude) for the transition in a particular molecule $\xi$ is thus written as

$$
M_{f i}^{(\xi)}=M_{f i}^{(\xi, a)}+M_{f i}^{(\xi, b)}
$$

where $M_{f i}^{(\xi, a)}$ is the matrix element for graph (a) of Fig. 2 and $M_{f i}^{(\xi, b)}$ that for graph (b). By well-established methods [23] these quantities can be written as

$$
\begin{aligned}
M_{f i}^{(\xi, a)}= & \left(\frac{\hbar c k}{4 \varepsilon_{0} V}\right)\left[\left\langle m^{\prime}\right\rangle(\langle m\rangle-1)\right]^{1 / 2} \alpha_{(i j)}^{10}(\omega, \omega) \\
& \times e_{i}^{(2)} e_{j}^{(2)} e^{-i 2 \mathbf{k} \cdot \mathbf{R}_{\xi}}
\end{aligned}
$$

and

$$
M_{f i}^{(\xi, b)}=-i\left(\frac{\hbar c k^{\prime}}{2 \varepsilon_{0} V}\right)^{1 / 2}\langle p\rangle^{1 / 2} \mu_{i}^{10} e_{i}^{(3)} e^{i \mathbf{k}^{\prime} \cdot \mathbf{R}_{\xi}}
$$

In these equations the position of the molecule is described by the vector $\mathbf{R}_{\xi}$, the wave vectors of the two beams of mode $\mathbf{m}_{1}$ and $\mathbf{m}_{2}$ are $\mathbf{k}$ and $\mathbf{k}^{\prime}$, respectively, $\mathbf{e}^{(n)}$ is a unit 
vector describing the polarization state of mode $\mathbf{m}_{n},\langle m\rangle$ and $\langle p\rangle$ are the mean numbers of photons in modes $\mathbf{m}_{2}$ and $\mathbf{m}_{3}$, and a repeated Cartesian index implies three-dimensional summation over that index. In deriving Eqs. (2) and (3) the state vectors describing the radiation fields have been assumed to be coherent laser states and so $\langle m\rangle$ $=\left\langle\alpha^{(2)}|\hat{n}| \alpha^{(2)}\right\rangle$, where $\left|\alpha^{(2)}\right\rangle$ is the coherent state representing mode 2 and $\hat{n}$ is the number operator. A similar expression may be written for $\langle p\rangle$. Also, the molecular parameters apparent in Eqs. (2) and (3) are the transition dipole $\mu_{i}^{10}$ $=\left\langle 1\left|\mu_{i}^{(\xi)}\right| 0\right\rangle$ and the index-symmetric transition polarizability:

$$
\alpha_{(i j)}^{10}(\omega, \omega)=\sum_{r}\left\{\frac{\mu_{i}^{1 r} \mu_{j}^{r 0}+\mu_{j}^{1 r} \mu_{i}^{r 0}}{\widetilde{E}_{r 0}-\hbar \omega}\right\},
$$

in which the complex energy term in the denominator takes the form $\widetilde{E}_{r 0}=E_{r}-E_{0}-i \Gamma_{r}$ to account for the damping associated with $\Gamma_{r}$, the linewidth of the excited state $|r\rangle$. The convention adopted here is to make the sign of the damping term negative to ensure compliance with time-reversal principles [20]. Introducing the density of states for the writing process, $\rho_{F}^{(1)}$, the rate at which the excited state is populated is given by Fermi's golden rule and clearly three contributions are apparent:

$$
\Gamma=\frac{2 \pi \rho_{F}^{(1)}}{\hbar}\left|M_{f i}^{(\xi)}\right|^{2}=\Gamma_{1}+\Gamma_{2}+\Gamma_{3}
$$

where

$$
\begin{aligned}
\Gamma_{1}= & \left.\frac{2 \pi \rho_{F}^{(1)}}{\hbar}\left(\frac{\hbar c k}{4 \varepsilon_{0} V}\right)^{2}[\langle m\rangle(\langle m\rangle-1)] \alpha_{(i j)}^{10} e_{i}^{(2)} e_{j}^{(2)}\right|^{2}, \\
\Gamma_{2}= & \frac{2 \pi \rho_{F}^{(1)}}{\hbar}\left\{i\left(\frac{\hbar c k}{4 \varepsilon_{0} V}\right)\left(\frac{\hbar c k^{\prime}}{2 \varepsilon_{0} V}\right)^{1 / 2}[\langle p\rangle\langle m\rangle(\langle m\rangle-1)]^{1 / 2}\right. \\
& \times \alpha_{(i j)}^{10} \bar{\mu}_{k}^{10} e_{i}^{(2)} e_{j}^{(2)} \bar{e}_{k}^{(3)} e^{\left.-i\left(2 \mathbf{k}+\mathbf{k}^{\prime}\right) \cdot \mathbf{R}_{\xi}+\text { c.c. }\right\}}
\end{aligned}
$$

and

$$
\Gamma_{3}=\frac{2 \pi \rho_{F}^{(1)}}{\hbar}\left(\frac{\hbar c k^{\prime}}{2 \varepsilon_{0} V}\right)\langle p\rangle\left|\mu_{i}^{10} e_{i}^{(3)}\right|^{2} .
$$

We see that the rate at which the excited state is populated depends on the position of the molecule, through $\Gamma_{2}$-and also on the molecular orientation, through the molecular matrix elements. It is this $\Gamma_{2}$ term that produces the grating within the sample. We note here that the periodicity of the grating, determined by $e^{-i\left(2 \mathbf{k}+\mathbf{k}^{\prime}\right) \cdot \mathbf{R}_{\xi}}$, is exactly that required for phase-matched second-harmonic generation from the probe beam, the signal being created in precisely the opposite direction to the harmonic pump beam.

Thus far we have a rate $\Gamma\left(\mathbf{R}_{\xi}\right)$ at which the upper state is populated during application of the two writing beams. If we take the effective time for which the beams are applied as $\Delta t$, the probability that the molecule $\xi$ is excited immedi- (a)

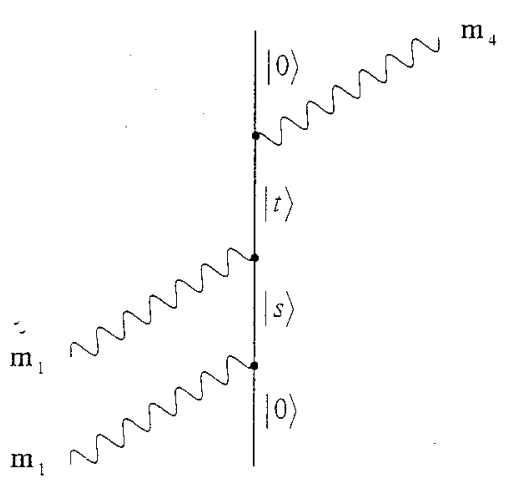

(b)

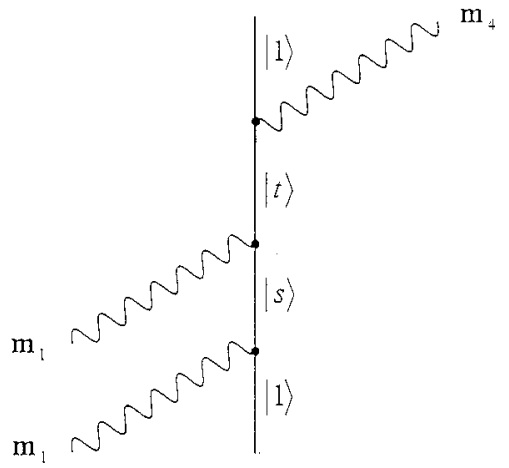

FIG. 3. Representative Feynman diagrams describing harmonic formation from molecules in (a) the ground electronic state and (b) the excited state.

ately after the pulses have passed is $P\left(\mathbf{R}_{\xi}\right)=\Gamma\left(\mathbf{R}_{\xi}\right) \Delta t$. The probe pulse arrives after a delay of $\tau(>\Delta t) \mathrm{sec}$, during which time the molecule, if excited, may relax. We suppose that it relaxes to the ground state via a simple exponential decay. At time $\tau$ the probability that the molecule is excited is hence

$$
P\left(\mathbf{R}_{\xi}, \tau\right)=\Gamma\left(\mathbf{R}_{\xi}\right) \Delta t e^{-k_{10}(\tau-\Delta t)},
$$

where $k_{10}$ is the decay constant.

\section{B. Clamped-molecule model}

We ignore for the present any movement (rotational or translational) that may occur in-between pulses, for that is a feature we accommodate later. The probe pulse encounters the associated population distribution in the sample and second-harmonic generation ( $\mathrm{SHG}$ ) is produced from it. Again, two possibilities arise, as illustrated in Fig. 3 (in which only the dominant of three contributory time orderings is shown). Writing as $M_{f i}^{\prime(\xi, a)}$ and $M_{f i}^{\prime(\xi, b)}$ the quantum matrix elements for these component processes, the effective matrix element for harmonic production will be as follows:

$$
M_{f i}^{\prime(\xi)}=\left[1-P\left(\mathbf{R}_{\xi}, \tau\right)\right] M_{f i}^{\prime(\xi, a)}+P\left(\mathbf{R}_{\xi}, \tau\right) M_{f i}^{\prime(\xi, b)},
$$

reflecting a statistical weighting of the appropriate quantum amplitudes. For a two-level system Eq. (10) is exact, and 
follows from use of the completeness relation for the molecular states. The two components involved in the harmonic generation process are in fact identical in terms of the photonics, differing only in their molecular mediation, and so we have

$$
\begin{aligned}
M_{f i}^{\prime(\xi, a)}= & -i\left(\frac{\hbar c k}{2 \varepsilon_{0} V}\right)\left(\frac{\hbar c k^{\prime}}{2 \varepsilon_{0} V}\right)^{1 / 2}[\langle n\rangle(\langle n\rangle-1)]^{1 / 2} \\
& \times \beta_{i(j k)} \bar{e}_{i}^{(4)} e_{j}^{(1)} e_{k}^{(1)} e^{i\left(2 \mathbf{k}+\mathbf{k}^{\prime}\right) \cdot \mathbf{R}_{\xi}}
\end{aligned}
$$

and

$$
\begin{aligned}
M_{f i}^{\prime(\xi, b)}= & -i\left(\frac{\hbar c k}{2 \varepsilon_{0} V}\right)\left(\frac{\hbar c k^{\prime}}{2 \varepsilon_{0} V}\right)^{1 / 2}[\langle n\rangle(\langle n\rangle-1)]^{1 / 2} \\
& \times \beta_{i(j k)}^{\prime} \bar{e}_{i}^{(4)} e_{j}^{(1)} e_{k}^{(1)} e^{i\left(2 \mathbf{k}+\mathbf{k}^{\prime}\right) \cdot \mathbf{R}_{\xi}}
\end{aligned}
$$

Here, $\langle n\rangle$ is the mean number of photons in mode 1 and an overbar represents complex conjugation. The indexsymmetric hyperpolarizabilities are given by

$$
\begin{aligned}
\beta_{i(j k)}= & \frac{1}{2} \sum_{s, t}\left[\frac{\mu_{i}^{0 t} \mu_{j}^{t s} \mu_{k}^{s 0}}{\left(\widetilde{E}_{t 0}-2 \hbar \omega\right)\left(\widetilde{E}_{s 0}-\hbar \omega\right)}\right. \\
& +\frac{\mu_{j}^{0 t} \mu_{i}^{t s} \mu_{k}^{s 0}}{\left(\widetilde{E}_{t 0}+\hbar \omega\right)\left(\widetilde{E}_{s 0}-\hbar \omega\right)}+\frac{\mu_{j}^{0 t} \mu_{k}^{t s} \mu_{i}^{s 0}}{\left(\widetilde{E}_{t 0}+\hbar \omega\right)\left(\widetilde{E}_{s 0}+2 \hbar \omega\right)} \\
& +\frac{\mu_{i}^{0 t} \mu_{k}^{t s} \mu_{j}^{s 0}}{\left(\widetilde{E}_{t 0}-2 \hbar \omega\right)\left(\widetilde{E}_{s 0}-\hbar \omega\right)}+\frac{\mu_{k}^{0 t} \mu_{i}^{t s} \mu_{j}^{s 0}}{\left(\widetilde{E}_{t 0}+\hbar \omega\right)\left(\widetilde{E}_{s 0}-\hbar \omega\right)} \\
& \left.+\frac{\mu_{k}^{0 t} \mu_{j}^{t s} \mu_{i}^{s 0}}{\left(\widetilde{E}_{t 0}+\hbar \omega\right)\left(\widetilde{E}_{s 0}+2 \hbar \omega\right)}\right]
\end{aligned}
$$

and

$$
\begin{aligned}
\beta_{i(j k)}^{\prime}= & \frac{1}{2} \sum_{s, t}\left[\frac{\mu_{i}^{1 t} \mu_{j}^{t s} \mu_{k}^{s 1}}{\left(\widetilde{E}_{t 1}-2 \hbar \omega\right)\left(\widetilde{E}_{s 1}-\hbar \omega\right)}\right. \\
& +\frac{\mu_{j}^{1 t} \mu_{i}^{t s} \mu_{k}^{s 1}}{\left(\widetilde{E}_{t 1}+\hbar \omega\right)\left(\widetilde{E}_{s 1}-\hbar \omega\right)}+\frac{\mu_{j}^{1 t} \mu_{k}^{t s} \mu_{i}^{s 1}}{\left(\widetilde{E}_{t 1}+\hbar \omega\right)\left(\widetilde{E}_{s 1}+2 \hbar \omega\right)} \\
& +\frac{\mu_{i}^{1 t} \mu_{k}^{t s} \mu_{j}^{s 1}}{\left(\widetilde{E}_{t 1}-2 \hbar \omega\right)\left(\widetilde{E}_{s 1}-\hbar \omega\right)}+\frac{\mu_{k}^{1 t} \mu_{i}^{t s} \mu_{j}^{s 1}}{\left(\widetilde{E}_{t 1}+\hbar \omega\right)\left(\widetilde{E}_{s 1}-\hbar \omega\right)} \\
& \left.+\frac{\mu_{k}^{1 t} \mu_{j}^{t s} \mu_{i}^{s 1}}{\left(\widetilde{E}_{t 1}+\hbar \omega\right)\left(\widetilde{E}_{s 1}+2 \hbar \omega\right)}\right],
\end{aligned}
$$

respectively. The total matrix element for SHG from the ensemble is thus

$$
\begin{aligned}
M_{f i}^{\prime}= & -i\left(\frac{\hbar c k}{2 \varepsilon_{0} V}\right)\left(\frac{\hbar c k^{\prime}}{2 \varepsilon_{0} V}\right)^{1 / 2}[\langle n\rangle(\langle n\rangle-1)]^{1 / 2} \\
& \times \bar{e}_{i}^{(4)} e_{j}^{(1)} e_{k}^{(1)} \sum_{\xi}\left\{\beta_{i(j k)}+P\left(\mathbf{R}_{\xi}, \tau\right) \Delta \beta_{i(j k)}\right\} \\
& \times e^{i\left(2 k+k^{\prime}\right) \cdot \mathbf{R}_{\xi}}
\end{aligned}
$$

where the hyperpolarizability difference between the upper and lower states has been written

$$
\Delta \beta_{i(j k)}=\beta_{i(j k)}^{\prime}-\beta_{i(j k)} .
$$

The rate of production of SHG from the ensemble is now given by the Fermi rule:

$$
R=\frac{2 \pi \rho_{F}^{(2)}}{\hbar}\left|\sum_{\xi} M_{f i}^{\prime(\xi)}\right|^{2}
$$

where $\rho_{F}^{(2)}$ is the density of states for the second (reading) process. Taking an orientational average and effecting the usual split into incoherent (single site) and coherent (multisite interference) terms, we have

$$
\langle R\rangle=\frac{2 \pi \rho_{F}^{(2)}}{\hbar}\left\langle\sum_{\xi}\left|M_{f i}^{\prime(\xi)}\right|^{2}+\sum_{\xi=\xi^{\prime}} M_{f i}^{\prime(\xi)} \bar{M}_{f i}^{\prime\left(\xi^{\prime}\right)}\right\rangle .
$$

The dominant contribution to SHG is hence the coherent term,

$$
R_{\mathrm{coh}}=\frac{2 \pi \rho_{F}^{(2)}}{\hbar} \sum_{\xi=\xi^{\prime}}\left\langle M_{f i}^{\prime(\xi)}\right\rangle\left\langle\bar{M}_{f i}^{\prime\left(\xi^{\prime}\right)}\right\rangle
$$

where we have assumed that differing molecules in the solution are orientationally uncorrelated, as is the case for the majority of pairs in the system. For any particular molecule we have

$$
\begin{aligned}
\left\langle M_{f i}^{\prime(\xi)}\right\rangle= & -i\left(\frac{\hbar c k}{2 \varepsilon_{0} V}\right)\left(\frac{\hbar c k^{\prime}}{2 \varepsilon_{0} V}\right)^{1 / 2}[\langle n\rangle(\langle n\rangle-1)]^{1 / 2} \bar{e}_{i}^{(4)} e_{j}^{(1)} e_{k}^{(1)} \\
& \times\left\langle\left\{\beta_{i(j k)}+P\left(\mathbf{R}_{\xi}, \tau\right) \Delta \beta_{i(j k)}\right\}\right\rangle e^{i\left(2 \mathbf{k}+\mathbf{k}^{\prime}\right) \cdot \mathbf{R}_{\xi}}
\end{aligned}
$$

Effecting the orientational average on the first term within braces in Eq. (18) leads to its disappearance, as is usual for SHG in isotropic media. The second term, however, contains " 'hidden" orientational factors through $P\left(\mathbf{R}_{\xi}, \tau\right)$, as a result of which the average is nonzero.

Using Eq. (9) we thus have

$$
\begin{aligned}
& \left\langle\Gamma\left(\mathbf{R}_{\xi}\right) \Delta t e^{-k_{10}(\tau-\Delta t)} \Delta \beta_{i(j k)}\right\rangle \\
& \quad=\left\langle\left(\Gamma_{1}+\Gamma_{2}+\Gamma_{3}\right) \Delta \beta_{i(j k)}\right\rangle \Delta t e^{-k_{10}(\tau-\Delta t)} .
\end{aligned}
$$

Of the three contributory terms, it is the middle one that will be responsible for the observed signal as it is the only one to exhibit the necessary phase matching when inserted into Eq. (18). We thus ignore the other two terms in Eq. (19). The correctness of this assumption is readily verified from the 
fact that the signal is not observed if either of the writing beams is blocked. Using the $\Gamma_{2}$ term in Eq. (19) we now find

$$
\begin{aligned}
\left\langle\Gamma\left(\mathbf{R}_{\xi}\right) \Delta t e^{-k_{10}(\tau-\Delta t)} \Delta \beta_{i(j k)}\right\rangle & \\
= & i \frac{2 \pi \rho_{F}^{(1)}}{\hbar}\left(\frac{\hbar c k}{4 \varepsilon_{0} V}\right)\left(\frac{\hbar c k^{\prime}}{2 \varepsilon_{0} V}\right)^{1 / 2}[\langle p\rangle\langle m\rangle(\langle m\rangle-1)]^{1 / 2} \\
& \times\left\langle\left\{\alpha_{(l m)}^{10} \bar{\mu}_{n}^{10} e_{l}^{(2)} e_{m}^{(2)} \bar{e}_{n}^{(3)} e^{\left.-i\left(2 \mathbf{k}+\mathbf{k}^{\prime}\right) \cdot \mathbf{R}_{\xi}+\text { c.c. }\right\}}\right.\right. \\
& \left.\times \Delta \beta_{i(j k)}\right\rangle \Delta t e^{-k_{10}(\tau-\Delta t)}
\end{aligned}
$$

with the exponential explicitly exhibiting the phase matching (and the complex conjugate term accounting for the fact that SHG can be produced from a fundamental beam propagating in the opposite direction, as also observed experimentally). The phase-matched, orientationally averaged matrix element is hence

$$
\begin{aligned}
\left\langle M_{f i}^{\prime(\xi)}\right\rangle= & \frac{\pi \rho_{F}^{(1)}}{\hbar}\left(\frac{\hbar c k}{2 \varepsilon_{0} V}\right)^{2}\left(\frac{\hbar c k^{\prime}}{2 \varepsilon_{0} V}\right)[\langle p\rangle\langle m\rangle(\langle m\rangle-1) \\
& \times\langle n\rangle(\langle n\rangle-1)]^{1 / 2}\left\langle\alpha_{(l m)}^{10} \bar{\mu}_{n}^{10} \Delta \beta_{i(j k)}\right\rangle \\
& \times e_{l}^{(2)} e_{m}^{(2)} \bar{e}_{n}^{(3)} \bar{e}_{i}^{(4)} e_{j}^{(1)} e_{k}^{(1)} \Delta t e^{-k_{10}(\tau-\Delta t)},
\end{aligned}
$$

which is necessarily position independent so that the phasematching double sum in Eq. (17) can be evaluated for the ensemble of $N$ molecules as $N(N-1) \approx N^{2}$ for large $N$. The resulting rate of SHG production is

$$
\begin{aligned}
R_{\mathrm{coh}}= & \frac{2 \pi^{3}\left(\rho_{F}^{(1)}\right)^{2} \rho_{F}^{(2)}}{\hbar^{3}}(N \Delta t)^{2}\left(\frac{\hbar c k}{2 \varepsilon_{0} V}\right)^{4}\left(\frac{\hbar c k^{\prime}}{2 \varepsilon_{0} V}\right) \\
& \times[\langle p\rangle\langle m\rangle(\langle m\rangle-1)\langle n\rangle(\langle n\rangle-1)] \\
& \times\left|\left\langle\alpha_{(l m)}^{10} \bar{\mu}_{n}^{10} \Delta \beta_{i(j k)}\right\rangle e_{l}^{(2)} e_{m}^{(2)} \bar{e}_{n}^{(3)} \bar{e}_{i}^{(4)} e_{j}^{(1)} e_{k}^{(1)}\right|^{2} \\
& \times e^{-2 k_{10}(\tau-\Delta t)}
\end{aligned}
$$

Casting the result in terms of the mean intensities of the beams, the final expression for the coherent SHG from the grating may be written

$$
\begin{aligned}
I_{\mathrm{sig}}^{(2 \omega)}= & \frac{\left(\rho_{F}^{(1)}\right)^{2}\left(k^{\prime}\right)^{3} g_{1}^{(2)} g_{2}^{(2)}(N \Delta t)^{2}}{256 \hbar^{2} c^{2} \varepsilon_{0}^{6}}\left(I_{1}^{(\omega)} I_{2}^{(\omega)}\right)^{2} I_{3}^{(2 \omega)} \\
& \times\left|\left\langle\alpha_{(l m)}^{10} \bar{\mu}_{n}^{10} \Delta \beta_{i(j k)}\right\rangle e_{l}^{(2)} e_{m}^{(2)} \bar{e}_{n}^{(3)} \bar{e}_{i}^{(4)} e_{j}^{(1)} e_{k}^{(1)}\right|^{2} \\
& \times e^{-2 k_{10}(\tau-\Delta t)}
\end{aligned}
$$

where $I_{n}^{(\omega)}$ is the mean intensity of the $n$th beam of frequency $\omega$, and $g_{n}^{(2)}$ is its degree of second-order coherence. Equation (22) thus exhibits the expected (and observed [5,6]) dependence on the intensities of the three input beams (quadratic with respect to the two fundamental beams and linear in the harmonic writing beam) and also the sample density $\left(I_{\text {sig }}^{(2 \omega)} \propto N^{2}\right)$. Dynamically this equation yields a simple exponential decay due to relaxation of the molecules from the excited to ground state- the lifetime of the decay therefore governed by the intrinsic fluorescence lifetime.
The polarization dependence of Eq. (22) is exactly that found previously for the case of coincident pulses [16]. An interesting feature of the result is its dependence on molecular polarizabilities. Evaluating the sixth-rank average we find

$$
\begin{aligned}
I_{\mathrm{sig}}^{(2 \omega)}= & \frac{\left(\rho_{F}^{(1)}\right)^{2}\left(k^{\prime}\right)^{3} g_{1}^{(2)} g_{2}^{(2)}(N \Delta t)^{2}}{256 \hbar^{2} c^{5} \varepsilon_{0}^{6}}\left(I_{1}^{(\omega)} I_{2}^{(\omega)}\right)^{2} I_{3}^{(2 \omega)} \\
& \times\left|\sum_{n=1}^{6} \Omega_{n} E_{n}\right|^{2} e^{-2 k_{10}(\tau-\Delta t)}
\end{aligned}
$$

where the linear matrix $\Omega$ comprises molecular parameters defined by

$$
\left[\begin{array}{l}
\Omega_{1} \\
\Omega_{2} \\
\Omega_{3} \\
\Omega_{4} \\
\Omega_{5} \\
\Omega_{6}
\end{array}\right]=\mathbf{A}\left[\begin{array}{c}
\bar{\mu}_{\gamma}^{10} \alpha_{(\beta \beta)}^{10} \Delta \beta_{\gamma(\alpha \alpha)} \\
\bar{\mu}_{\beta}^{10} \alpha_{(\beta \gamma)}^{10} \Delta \beta_{\gamma(\alpha \alpha)} \\
\bar{\mu}_{\gamma}^{10} \alpha_{(\alpha \beta)}^{10} \Delta \beta_{\gamma(\alpha \beta)} \\
\bar{\mu}_{\beta}^{10} \alpha_{(\alpha \gamma)}^{10} \Delta \beta_{\gamma(\alpha \beta)} \\
\bar{\mu}_{\gamma}^{10} \alpha_{(\alpha \gamma)}^{10} \Delta \beta_{\beta(\alpha \beta)} \\
\bar{\mu}_{a}^{10} \alpha_{(\gamma \gamma)}^{10} \Delta \beta_{\beta(\alpha \beta)}
\end{array}\right]
$$

the matrix of coefficients being given by

$$
\mathbf{A}=\frac{1}{105}\left[\begin{array}{cccccc}
8 & -5 & -5 & 4 & 4 & -5 \\
-5 & 11 & 4 & -6 & -6 & 4 \\
-5 & 4 & 11 & -6 & -6 & 4 \\
4 & -6 & -6 & 16 & 2 & -6 \\
4 & -6 & -6 & 2 & 16 & -6 \\
-5 & 4 & 4 & -6 & -6 & 11
\end{array}\right]
$$

and the linear matrix $\mathbf{E}$ embodies a set of six, in general linearly independent, polarization parameters

$$
\begin{gathered}
E_{1}=\left(\mathbf{e}_{1} \cdot \mathbf{e}_{1}\right)\left(\mathbf{e}_{2} \cdot \mathbf{e}_{2}\right)\left(\overline{\mathbf{e}}_{3} \cdot \overline{\mathbf{e}}_{4}\right), \quad E_{2}=\left(\mathbf{e}_{1} \cdot \mathbf{e}_{1}\right)\left(\mathbf{e}_{2} \cdot \overline{\mathbf{e}}_{3}\right)\left(\mathbf{e}_{2} \cdot \overline{\mathbf{e}}_{4}\right), \\
E_{3}=\left(\mathbf{e}_{1} \cdot \mathbf{e}_{2}\right)^{2}\left(\overline{\mathbf{e}}_{3} \cdot \overline{\mathbf{e}}_{4}\right), \quad E_{4}=\left(\mathbf{e}_{1} \cdot \mathbf{e}_{2}\right)\left(\mathbf{e}_{1} \cdot \overline{\mathbf{e}}_{3}\right)\left(\mathbf{e}_{2} \cdot \overline{\mathbf{e}}_{4}\right), \\
E_{5}=\left(\mathbf{e}_{1} \cdot \mathbf{e}_{2}\right)\left(\mathbf{e}_{1} \cdot \overline{\mathbf{e}}_{4}\right)\left(\mathbf{e}_{2} \cdot \overline{\mathbf{e}}_{3}\right), \quad E_{6}=\left(\mathbf{e}_{1} \cdot \overline{\mathbf{e}}_{3}\right)\left(\mathbf{e}_{2} \cdot \mathbf{e}_{2}\right)\left(\mathbf{e}_{1} \cdot \overline{\mathbf{e}}_{4}\right) .
\end{gathered}
$$

We note that in Eq. (24) we have used Greek indices to denote a tensor component written in terms of the molecular axes, Latin indices now being reserved for components in the laboratory fixed frame. The polarization characteristics may now be determined and should be identical in form to those of the coincident-pulse case. However, one would not expect them to be exactly the same because of their different dependence on molecular properties.

\section{Effects of molecular motion}

The dynamic response predicted by the clamped-molecule model is a simple exponential. To account for more complex dynamics observed experimentally $[5,6]$ the model can now be refined to encompass molecular motion. At the instant in time when the first pair of pulses excites the molecule, let us denote the position as $\mathbf{R}_{\xi}^{(0)}$ and also let $\boldsymbol{\Omega}_{\xi}^{(0)}$ represent a set 
of three orientation coordinates, reflecting the angular disposition of the molecule against a laboratory-fixed frame. The probability of excitation during application of the writing beams is hence $P\left(\mathbf{R}_{\xi}^{(0)}\right)=\Gamma\left(\mathbf{R}_{\xi}^{(0)}, \Omega_{\xi}^{(0)}\right) \Delta t$, assuming there is no significant molecular motion within the write interval. At a later time $\tau$, the probability that the molecule, initially at position $\mathbf{R}_{\xi}^{(0)}$, is still excited is thus

$$
P\left(\mathbf{R}_{\xi}^{(0)}, \tau\right)=\Gamma\left(\mathbf{R}_{\xi}^{(0)}, \Omega_{\xi}^{(0)}\right) \Delta t e^{-k_{10}(\tau-\Delta t)} .
$$

However its new position and orientation at this time may be represented as $\left(\mathbf{R}_{\xi}, \Omega_{\xi}\right)$, so that the corresponding molecular matrix element for SHG is

$$
\begin{aligned}
M_{f i}^{\prime(\xi)}= & -i\left(\frac{\hbar c k}{2 \varepsilon_{0} V}\right)\left(\frac{\hbar c k^{\prime}}{2 \varepsilon_{0} V}\right)^{1 / 2}[\langle n\rangle(\langle n\rangle-1)]^{1 / 2} \bar{e}_{i}^{(4)} e_{j}^{(1)} e_{k}^{(1)} \\
& \times\left\{\beta_{i(j k)}^{(\xi)}\left(\Omega_{\xi}\right)+P\left(\mathbf{R}_{\xi}^{(0)}, \tau\right) \Delta \beta_{i(j k)}\left(\Omega_{\xi}\right)\right\} \\
& \times e^{i\left(2 \mathbf{k}+\mathbf{k}^{\prime}\right) \cdot \mathbf{R}_{\xi}}
\end{aligned}
$$

When the ensemble average is effected, the first term disappears as usual and, retaining only the $\Gamma_{2}$ term (evaluated at $\left.\mathbf{R}_{\xi}^{(0)}\right)$ as before, we have

$$
\begin{aligned}
\left\langle M_{f i}^{(\xi)}\right\rangle= & -i\left(\frac{\hbar c k}{2 \varepsilon_{0} V}\right)\left(\frac{\hbar c k^{\prime}}{2 \varepsilon_{0} V}\right)^{1 / 2}[\langle n\rangle(\langle n\rangle-1)]^{1 / 2} \\
& \times \bar{e}_{i}^{(4)} e_{j}^{(1)} e_{k}^{(1)}\left\langle\Gamma_{2}\left(\mathbf{R}_{\xi}^{(0)}, \Omega_{\xi}^{(0)}\right) \Delta \beta_{i(j k)}\left(\Omega_{\xi}\right)\right\rangle \\
& \times e^{i\left(2 \mathbf{k}+\mathbf{k}^{\prime}\right) \cdot \mathbf{R}_{\xi} \Delta t e^{-k_{10}(\tau-\Delta t)}}
\end{aligned}
$$

Taking the quasi-phase-matched term, this yields

$$
\begin{aligned}
R_{\mathrm{coh}}= & \frac{\pi \rho_{F}^{(1)}}{\hbar}\left(\frac{\hbar c k}{2 \varepsilon_{0} V}\right)^{2}\left(\frac{\hbar c k^{\prime}}{2 \varepsilon_{0} V}\right)[\langle p\rangle\langle m\rangle(\langle m\rangle-1)\langle n\rangle \\
& \times(\langle n\rangle-1)]^{1 / 2} e_{l}^{(2)} e_{m}^{(2)} \bar{e}_{n}^{(3)} \bar{e}_{i}^{(4)} e_{j}^{(1)} e_{k}^{(1)} \\
& \times \Delta t e^{-k_{10}(\tau-\Delta t)}\left\langle\alpha_{l(m)}^{10}\left(\Omega_{\xi}^{(0)}\right) \bar{\mu}_{n}^{10}\left(\Omega_{\xi}^{(0)}\right)\right. \\
& \left.\times \Delta \beta_{i(j k)}\left(\Omega_{\xi}\right)\right\rangle e^{i\left(2 \mathbf{k}+\mathbf{k}^{\prime}\right) \cdot\left(\mathbf{R}_{\xi}-\mathbf{R}_{\xi}^{(0)}\right)} .
\end{aligned}
$$

The effects of translation and rotation are apparent in this expression. However, taking a typical diffusion coefficient of $D \sim 10^{-9} \mathrm{~m}^{2} \mathrm{~s}^{-1}$ we can estimate the mean distance travelled in $100 \mathrm{ps}$ as $\langle x\rangle \sim 2(D t / \pi)^{1 / 2} \sim 3.6 \times 10^{-10} \mathrm{~m}$. Even for motion exactly collinear with the wave-vector mismatch, the scalar product $\left(2 \mathbf{k}+\mathbf{k}^{\prime}\right) \cdot\left(\mathbf{R}_{\xi}-\mathbf{R}_{\xi}^{(0)}\right) \sim 3 \times 10^{-3}$, where $\lambda$ $=800 \mathrm{~nm}$ and a refractive index difference of $\Delta n \sim 0.5$ has been assumed. For other angles the value of the scalar product will be still smaller. From this simple calculation it is immediately evident that on the ps timescale we can ignore translational diffusion. This makes the right-hand side of Eq. (30) position independent, so that the phase-matching double sum can be carried out as usual; the rate of coherent SHG production is then

$$
\begin{aligned}
R_{\mathrm{coh}}= & \frac{2 \pi^{3}\left(\rho_{F}^{(1)}\right)^{2} \rho_{F}^{(2)}}{\hbar^{3}}(N \Delta t)^{2}\left(\frac{\hbar c k}{2 \varepsilon_{0} V}\right)^{4}\left(\frac{\hbar c k}{2 \varepsilon_{0} V}\right)^{2} \\
& \times[\langle p\rangle\langle m\rangle(\langle m\rangle-1)\langle n\rangle(\langle n\rangle-1)] \\
& \times \mid\left\langle\alpha_{(l m)}^{10}\left(\Omega_{\xi}^{(0)}\right) \bar{\mu}_{n}^{10}\left(\Omega_{\xi}^{(0)}\right) \Delta \beta_{i(j k)}\left(\Omega_{\xi}\right)\right\rangle \\
& \times\left. e_{l}^{(2)} e_{m}^{(2)} \bar{e}_{n}^{(3)} \bar{e}_{i}^{(4)} e_{j}^{(1)} e_{k}^{(1)}\right|^{2} e^{-2 k_{10}(\tau-\Delta t)}
\end{aligned}
$$

Comparing Eqs. (31) and (21) we observe that the effect of molecular rotational motion can be accounted for by simply replacing the orientational average $\left\langle\alpha_{(l m)}^{10} \bar{\mu}_{n}^{10} \Delta \beta_{i(j k)}\right\rangle$ with $\left\langle\alpha_{(l m)}^{10}\left(\Omega_{\xi}^{(0)}\right) \bar{\mu}_{n}^{10}\left(\Omega_{\xi}^{(0)}\right) \Delta \beta_{i(j k)}\left(\Omega_{\xi}\right)\right\rangle$, which thereby correlates the properties of the molecule at the two times when pulses are present. As this correlation is time dependent, so too will be the average. The ensemble average is made at time $\tau$ by averaging over all the possible orientations of a molecule-fixed set of coordinates $(x, y, z)$, the tensorial components being given in terms of laboratory-fixed coordinates $(X, Y, Z)$. Introducing direction cosines $l_{\alpha i}$ between the molecular $\alpha$ axis and laboratory $i$ axis we have explicitly

$$
\begin{aligned}
\left\langle\alpha_{(l m)}^{10}\right. & \left.\left(\Omega_{\xi}^{(0)}\right) \bar{\mu}_{n}^{10}\left(\Omega_{\xi}^{(0)}\right) \Delta \beta_{i(j k)}\left(\Omega_{\xi}\right)\right\rangle \\
= & \alpha_{(\lambda \mu)}^{10} \bar{\mu}_{\nu}^{10} \Delta \beta_{\tilde{\alpha}(\tilde{\beta} \tilde{\gamma})}\left\langle l_{\tilde{\alpha} i}(\tau) l_{\tilde{\beta} j}(\tau) l_{\tilde{\gamma} k}(\tau) l_{\lambda l}(0)\right. \\
& \left.\times l_{\mu m}(0) l_{\nu n}(0)\right\rangle,
\end{aligned}
$$

where the polarizability components, fixed within the molecular frame (and therefore invariant upon rotation of this frame), have been removed from the average. In writing Eq. (32) we also introduced a tilde to refer to components of the molecular frame at time $\tau$.

The direction cosines at $\tau=0$ can now be related to those at $t=\tau$ using $l_{\lambda l}(0)=l_{\lambda \tilde{\delta}}(\tau) l_{\tilde{\delta} l}(\tau)$, where $\widetilde{\delta}$ refers to a component of the molecular frame at time $\tau$ and repetition of a Cartesian index implies summation. The $l_{\lambda} \tilde{\delta}(\tau)$ part comes out of the ensemble average because it relates to molecular axes only, the net result being

$$
\begin{aligned}
& \left\langle\alpha_{(l m)}^{10}\left(\Omega_{\tilde{\xi}}^{(0)}\right) \bar{\mu}_{n}^{10}\left(\Omega_{\tilde{\xi}}^{(0)}\right) \Delta \beta_{i(j k)}\left(\Omega_{\xi}\right)\right\rangle \\
& \quad=\alpha_{(\lambda \mu)}^{10} \bar{\mu}_{\nu}^{10} \Delta \beta_{\tilde{\alpha}(\tilde{\beta} \tilde{\gamma})}\left\langle l_{\tilde{\alpha} i} l_{\tilde{\beta} j} l_{\tilde{\gamma} k} l_{\tilde{\delta} l} l_{\tilde{\varepsilon} m} l_{\tilde{\phi} n}\right\rangle F_{(\lambda \tilde{\delta}, \mu \tilde{\varepsilon}, \nu \tilde{\phi})},
\end{aligned}
$$

where

$$
F_{(\lambda \tilde{\delta}, \mu \widetilde{\varepsilon}, \nu \tilde{\phi})}=l_{\lambda \tilde{\delta}}(\tau) l_{\mu \tilde{\varepsilon}}(\tau) l_{\nu \tilde{\phi}}(\tau)
$$

The tensor $\mathbf{F}$ contains all the time dependence in the righthand side of Eq. (33) because the isotropic average accommodates all possible molecular orientations and is thus independent of time. The indices in Eq. (34) are grouped in such a way as to emphasize the invariance with respect to interchange of any of the pairs of indices in parentheses, i.e., $F_{(\lambda \tilde{\delta}, \mu \tilde{\varepsilon}, \nu \tilde{\phi})}=F_{(\mu \widetilde{\varepsilon}, \lambda \tilde{\delta}, \nu \tilde{\phi})}$, etc. Inserting Eq. (33) into Eq. (31) and computing the isotropic average we have 
TABLE I. Eigenvalues and eigenvectors of the asymmetric rotor.

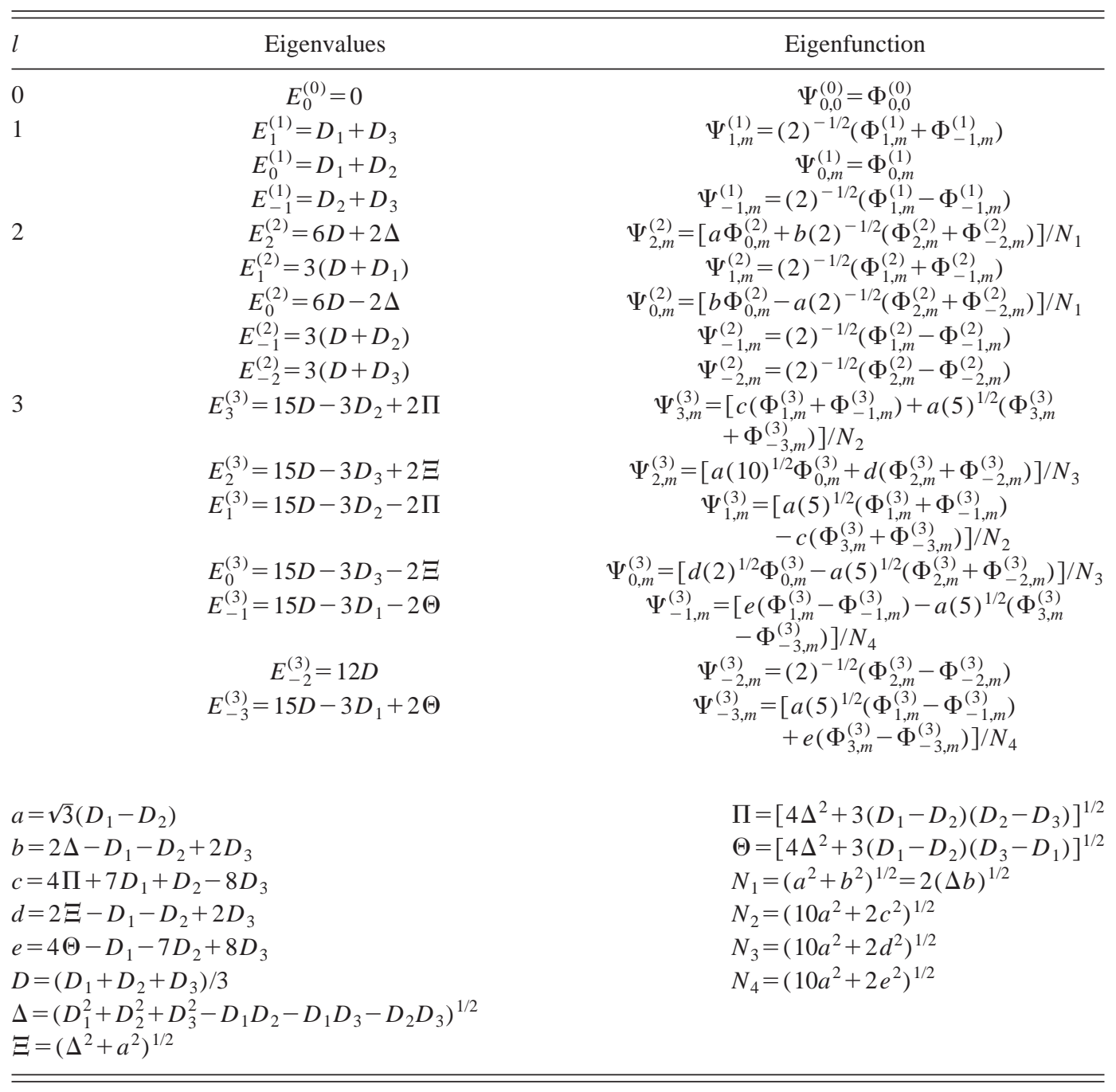

$$
\begin{aligned}
I_{\text {sig }}^{(2 \omega)}= & \frac{\left(\rho_{F}^{(1)}\right)^{2}\left(k^{\prime}\right)^{3} g_{1}^{(2)} g_{2}^{(2)}(N \Delta t)^{2}}{256 \hbar^{2} c^{5} \varepsilon_{0}^{6}}\left(I_{1}^{(\omega)} I_{2}^{(\omega)}\right)^{2} I_{3}^{(2 \omega)} \\
& \times\left|\sum_{n=1}^{6} \Lambda_{n} E_{n}\right|^{2} e^{-2 k_{10}(\tau-\Delta t)}
\end{aligned}
$$

with

$$
\left[\begin{array}{c}
\Lambda_{1} \\
\Lambda_{2} \\
\Lambda_{3} \\
\Lambda_{4} \\
\Lambda_{5} \\
\Lambda_{6}
\end{array}\right]=\mathbf{A}\left[\begin{array}{l}
\bar{\mu}_{\lambda}^{10} \alpha_{(\mu \nu)}^{10} \Delta \beta_{\tilde{\gamma}(\tilde{\alpha} \tilde{\alpha})} F_{(\lambda \tilde{\gamma}, \mu \tilde{\beta}, \nu \tilde{\beta})} \\
\bar{\mu}_{\lambda}^{10} \alpha_{(\mu \nu)}^{10} \Delta \beta_{\tilde{\gamma}(\tilde{\alpha} \tilde{\alpha})} F_{(\lambda \tilde{\beta}, \mu \tilde{\gamma}, \nu \tilde{\beta})} \\
\bar{\mu}_{\lambda}^{10} \alpha_{(\mu \nu)}^{10} \Delta \beta_{\tilde{\gamma}(\tilde{\alpha} \tilde{\beta})} F_{(\lambda \tilde{\gamma}, \mu \tilde{\alpha}, \nu \tilde{\beta})} \\
\bar{\mu}_{\lambda}^{10} \alpha_{(\mu \nu)}^{10} \Delta \beta_{\tilde{\gamma}(\tilde{\alpha} \tilde{\beta})} F_{(\lambda \tilde{\beta}, \mu \tilde{\gamma}, \nu \tilde{\alpha})} \\
\bar{\mu}_{\lambda}^{10} \alpha_{(\mu \nu)}^{10} \Delta \beta_{\tilde{\beta}(\tilde{\alpha} \tilde{\beta})} F_{(\lambda \tilde{\gamma}, \mu \tilde{\alpha}, \nu \tilde{\gamma})} \\
\bar{\mu}_{\lambda}^{10} \alpha_{(\mu \nu)}^{10} \Delta \beta_{\tilde{\beta}(\tilde{\alpha} \tilde{\beta})} F_{(\lambda \tilde{\alpha}, \mu \tilde{\gamma}, \nu \tilde{\gamma})}
\end{array}\right]
$$

and with $\mathbf{A}$ as given by Eq. (25). $\mathbf{F}(\tau)$ gives the additional time variation due to rotation of the molecules. We are interested here in molecules in a fluid host and so we shall model the rotational motion stochastically as a diffusion process. In this case the tensor takes the form

$$
F_{(\lambda \tilde{\delta}, \mu \tilde{\varepsilon}, \nu \tilde{\phi})}=\left\langle l_{\lambda \tilde{\delta}} l_{\mu \tilde{\varepsilon}} l_{\nu \tilde{\phi}}\right\rangle_{\mathrm{Rd}}
$$

where the subscript "R.d.' indicates an average taken over an ensemble of molecular frames rotating due to rotational diffusion dynamics.

Using a method first developed by Favro [18], we write the ensemble average of a general function of orientation and time, $g(\Omega, \tau)$, as

$$
\langle g\rangle_{\mathrm{Rd}}=\int g(\Omega, \tau) \rho(\Omega, \tau) \mathbf{d} \Omega
$$

where $\rho(\Omega, \tau) \mathbf{d} \Omega$ is the probability of finding a member of the ensemble of rotating frames oriented within the range $(\Omega, \Omega+\mathbf{d} \Omega)$ at time $\tau$. The distribution function obeys a differential equation analogous to the time-dependent Schrödinger equation

$$
\frac{\partial}{\partial \tau} \rho(\Omega, \tau)=-H \rho(\Omega, \tau),
$$

where 


$$
H=\sum_{i, j} L_{i} D_{i j} L_{j}
$$

with $\mathbf{D}$ the molecular diffusion tensor and $\mathbf{L}$ the quantummechanical angular momentum operator. If we use a set of coordinates that diagonalizes the diffusion tensor, as will henceforth be assumed, Eq. (40) may be written as

$$
H=\sum_{i} D_{i} L_{i}^{2}
$$

where $D_{1}, D_{2}$, and $D_{3}$ are the principal diffusion coefficients. The dynamics of the ensemble of molecular frames moving in response to rotational diffusion is thus identical to the quantum-mechanical problem of an asymmetric top, provided we identify $D_{i}$ with $\hbar^{2} / 2 I_{i}$, where $I_{i}$ is the corresponding moment of inertia. Equation (39) may be solved with a Green's function formulation, that is,

$$
\rho(\Omega, \tau)=\int \rho\left(\Omega_{0}, 0\right) G\left(\Omega_{0} \mid \Omega, \tau\right) d \Omega_{0} .
$$

Here $\rho\left(\Omega_{0}, 0\right)$ is the initial probability that the frame has orientation $\Omega_{0}$ and $G\left(\Omega_{0} \mid \Omega, \tau\right)$ is the Green's function describing the rotation of the frame from $\Omega_{0}$ at $t=0$ into $\Omega$ at time $t=\tau$. In our case we can take the initial ensemble of frames to have a common orientation, $\mathbf{0}$, say, so that $\rho\left(\Omega_{0}, 0\right)=\delta\left(\Omega_{0}\right)$, the Dirac $\delta$ function. This implies, using Eq. (42), that

$$
\rho(\Omega, \tau)=G(\mathbf{0} \mid \Omega, \tau) .
$$

This function is now expanded in terms of asymmetric rotor wave functions, $\Psi_{n}(\Omega)$, the solution to Eq. (39) being

$$
G(\mathbf{0} \mid \Omega, \tau)=\sum_{n} \Psi_{n}(0) \Psi_{n}(\Omega) \exp \left(-E_{n} \tau\right)
$$

with the initial condition

$$
G(\mathbf{0} \mid \Omega, 0)=\sum_{n} \bar{\Psi}_{n}(0) \Psi_{n}(\Omega)=\delta(\Omega)
$$

and where $E_{n}$ are the eigenvalues corresponding to $\Psi_{n}(\Omega)$. The latter wave functions can themselves be expressed in terms of symmetric rotor wave functions $\Phi_{k, m}^{(l)}(\Omega)$ :

$$
\Psi_{n}(\Omega)=\Psi_{\eta, m}^{(l)}(\Omega)=\sum_{k=-l}^{l} A_{\eta, k}^{(l)} \Phi_{k, m}^{(l)}(\Omega) .
$$

The coefficients $A_{\eta, k}^{(l)}$ and eigenvalues $E_{\eta}^{(l)}$ have been tabulated by Favro [18] and Huntress [24] for $l \leqslant 2$ - here, however, we require these quantities up to $l=3$. Calculation of the required values gives the results shown in Table I.

The symmetric rotor functions are orthonormal in the sense

$$
\int \Phi_{k^{\prime}, m^{\prime}}^{\left(l^{\prime}\right)}(\Omega) \Phi_{k, m}^{(l)}(\Omega) d \Omega=\delta_{l l^{\prime}} \delta_{k k^{\prime}} \delta_{m m^{\prime}},
$$

and are expressible in terms of Wigner rotation matrices, which describe the transformation from one set of coordinates to another by rotation through the Euler angles $\Omega$ $=(\alpha, \beta, \gamma)$ :

$$
\Phi_{k, m}^{(l)}(\Omega)=(-1)^{k-m}\left[(2 l+1) / 8 \pi^{2}\right]^{1 / 2} D_{k, m}^{(l)}(\Omega)
$$

Explicitly,

$$
\begin{aligned}
D_{k, m}^{(l)}(\Omega)= & \sum_{p}(-1)^{p} \frac{\sqrt{(l+m) !(l-m) !(l+k) !(l-k) !}}{p !(l-k-p) !(l+m-p) !(k-m+p) !} \\
& \times e^{i m \gamma}\left(\cos \frac{\beta}{2}\right)^{2 l+m-k-2 p}\left(\sin \frac{\beta}{2}\right)^{k-m+2 p} e^{i k \alpha},
\end{aligned}
$$

where the $p$ summation is taken over all integers. Using Eqs. (37), (38), (43)-(45), (47), and (48) we obtain

$$
F_{(\lambda \tilde{\delta}, \mu \tilde{\varepsilon}, \nu \tilde{\phi})}=\sum_{l, \eta} G_{(\lambda \tilde{\delta}, \mu \tilde{\varepsilon}, \nu \tilde{\phi})}^{(l ; \eta)} \exp -E_{\eta}^{(l)} \tau
$$

where

$$
G_{(\lambda \tilde{\delta}, \mu \tilde{\varepsilon}, \nu \tilde{\phi})}^{(l ; \eta)}=\frac{(2 l+1)}{8 \pi^{2}} \sum_{k, m=-l}^{l}(-1)^{m+k} A_{\eta, m}^{(l)} A_{\eta, k}^{(l)} F_{(\lambda \tilde{\delta}, \mu \tilde{\varepsilon}, \nu \tilde{\phi})}^{(l ; k, m)}
$$

and

$$
F_{(\lambda \tilde{\delta}, \mu \widetilde{\varepsilon}, \nu \tilde{\phi})}^{(l ; k, m)}=\int l_{\lambda} l_{\mu \widetilde{\varepsilon}} l_{\nu \tilde{\phi}} D_{k, m}^{(l)}(\Omega) d \Omega
$$

Associating the indices $x, y$, and $z$ with the numbers 1,2 , and 3 , respectively, the direction cosines may be written

$$
l_{\lambda \tilde{\delta}}=\sum_{i, j} M_{i j} \delta_{\lambda i} \delta_{\delta j}
$$

where the matrix $\mathbf{M}$ is given by 


$$
\mathbf{M}=\left[\begin{array}{ccc}
\cos \alpha \cos \beta \cos \gamma-\sin \alpha \sin \gamma & -\sin \alpha \cos \beta \cos \gamma-\cos \alpha \sin \gamma & \sin \beta \cos \gamma \\
\cos \alpha \cos \beta \sin \gamma+\sin \alpha \cos \gamma & -\sin \alpha \cos \beta \sin \gamma+\cos \alpha \cos \gamma & \sin \beta \sin \gamma \\
-\cos \alpha \sin \beta & \sin \alpha \sin \beta & \cos \beta
\end{array}\right] .
$$

As the Wigner matrices are complete we can then write

$$
l_{\lambda \tilde{\delta}}=\sum_{l, k, m} a_{\lambda \tilde{\delta}}^{(l, k, m)} D_{k, m}^{(l)}(\Omega)
$$

where

$$
a_{\lambda \tilde{\delta}}^{(l, k, m)}=\frac{2 l+1}{8 \pi^{2}} \int l_{\lambda} \bar{\delta}_{k, m}^{(l)}(\Omega) d \Omega .
$$

Inserting Eq. (52) into Eq. (55) we have

$$
a_{\lambda \tilde{\delta}}^{(l, k, m)}=\frac{2 l+1}{8 \pi^{2}} \sum_{i, j} \int M_{i j} \bar{D}_{k, m}^{(l)} d \Omega \delta_{\lambda l} \delta_{\tilde{\delta} j} .
$$

Each integral here can be written in the form

$$
\int M_{i j} \bar{D}_{k, m}^{(l)} d \Omega=\frac{4 \pi^{2}}{(l+2) !}\left(\begin{array}{c}
\delta_{k 1} \\
\delta_{k 0} \\
\delta_{k(-1)}
\end{array}\right)^{T} \mathbf{B}^{l, i j}\left(\begin{array}{c}
\delta_{m 1} \\
\delta_{m 0} \\
\delta_{m(-1)}
\end{array}\right) .
$$

where $\mathbf{B}^{l, i j}$ is a matrix of numbers dependent only on $l$. Thus we have from Eq. (56)

$$
a_{\lambda \widetilde{\delta}}^{(l, k, m)}=\frac{2 l+1}{(l+2) !}\left(\begin{array}{c}
\delta_{k 1} \\
\delta_{k 0} \\
\delta_{k(-1)}
\end{array}\right)^{T} \mathbf{C}_{\lambda \tilde{\delta}}^{(l)}\left(\begin{array}{c}
\delta_{m 1} \\
\delta_{m 0} \\
\delta_{m(-1)}
\end{array}\right)
$$

$$
\begin{aligned}
& =\frac{1}{2}\left(\begin{array}{c}
\delta_{k 1} \\
\delta_{k 0} \\
\delta_{k(-1)}
\end{array}\right)^{T} \mathbf{C}_{\lambda \tilde{\delta}}^{(l)}\left(\begin{array}{c}
\delta_{m 1} \\
\delta_{m 0} \\
\delta_{m(-1)}
\end{array}\right) \\
& =\frac{1}{2} \sum_{i, j=1}^{3}\left(C_{\lambda \tilde{\delta}}^{(l)}\right)_{i j} \delta_{k(2-i)} \delta_{m(2-j)}
\end{aligned}
$$

where the second equality follows from Eq. (59) which is obtained by direct computation:

$$
\begin{aligned}
\mathbf{C}_{\lambda \tilde{\delta}}^{(l)} & =\delta_{l 1}\left(\begin{array}{ccc}
\left(\delta_{\lambda x}-i \delta_{\lambda y}\right)\left(\delta_{\tilde{\delta} x}+i \delta_{\tilde{\delta} y}\right) & -\sqrt{2} \delta_{\lambda z}\left(\delta_{\tilde{\delta} x}+i \delta_{\tilde{\delta} y}\right) & -\left(\delta_{\lambda x}+i \delta_{\lambda y}\right)\left(\delta_{\tilde{\delta} x}+i \delta_{\tilde{\delta} y}\right) \\
-\sqrt{2}\left(\delta_{\lambda x}-i \delta_{\lambda y}\right) \delta_{\tilde{\delta} z} & 2 \delta_{\lambda z} \delta_{\tilde{\delta} z} & \sqrt{2}\left(\delta_{\lambda x}+i \delta_{\lambda y}\right) \delta_{\tilde{\delta} z} \\
-\left(\delta_{\lambda x}-i \delta_{\lambda y}\right)\left(\delta_{\tilde{\delta} x}-i \delta_{\tilde{\delta} y}\right) & \sqrt{2} \delta_{\lambda z}\left(\delta_{\tilde{\delta} x}-i \delta_{\tilde{\delta} y}\right) & \left(\delta_{\lambda x}+i \delta_{\lambda y}\right)\left(\delta_{\tilde{\delta} x}-i \delta_{\tilde{\delta} y}\right)
\end{array}\right) \\
& =\delta_{l 1} \Delta^{(\tilde{\lambda \tilde{\delta})}}
\end{aligned}
$$

The $\Delta^{(\lambda \tilde{\delta})}$ matrix defined here will shortly be shown to form the basis for our expressions for $F_{(\lambda \tilde{\delta}, \mu \tilde{\varepsilon}, \nu \tilde{\phi})}^{(l, k, m)}$. Some properties of this matrix are given below in Eqs. (60)-(63).

$$
\begin{aligned}
& \sum_{\lambda} \Delta^{(\lambda \lambda)}=2 \mathbf{U} \quad(\mathbf{U} \text { is the unit } 3 \times 3 \text { matrix }), \\
& \operatorname{Tr}\left(\Delta^{(\lambda \tilde{\delta})}\right)=2 \delta_{\lambda \tilde{\delta}}, \\
& \Delta_{i j}^{(\lambda \tilde{\delta})}=\bar{\Delta}_{j i}^{(\tilde{\delta} \lambda)}, \\
& \Delta_{11}^{(\lambda \tilde{\delta})}=\bar{\Delta}_{33}^{(\lambda \tilde{\delta})}, \quad \Delta_{22}^{(\lambda \tilde{\delta})}=\bar{\Delta}_{22}^{(\lambda \tilde{\delta})}, \\
& \Delta_{13}^{(\lambda \tilde{\delta})}=\bar{\Delta}_{31}^{(\lambda \tilde{\delta})}, \quad \Delta_{12}^{(\lambda \tilde{\delta})}=-\bar{\Delta}_{32}^{(\lambda \tilde{\delta})}, \quad \Delta_{21}^{(\lambda \tilde{\delta})}=-\bar{\Delta}_{23}^{(\lambda \tilde{\delta})} .
\end{aligned}
$$

From Eq. (51) and Eq. (54), we have

$$
\begin{aligned}
F_{(\lambda \tilde{\delta}, \mu \tilde{\varepsilon}, \nu \tilde{\phi})}^{(l ; k, m)}= & \sum_{l^{\prime}, k^{\prime}, m^{\prime}} \sum_{l^{\prime \prime}, k^{\prime \prime}, m^{\prime \prime}} \sum_{l^{\prime \prime \prime}, k^{\prime \prime \prime}, m^{\prime \prime \prime}} a_{\lambda \tilde{\delta}}^{\left(l^{\prime}, k^{\prime}, m^{\prime}\right)} a_{\mu \tilde{\varepsilon}}^{\left(l^{\prime \prime}, k^{\prime \prime}, m^{\prime \prime}\right)} \\
& \times a_{\nu \tilde{\phi}}^{\left(l^{\prime \prime \prime}, k^{\prime \prime \prime}, m^{\prime \prime \prime}\right)} \int D_{k, m}^{(l)}(\Omega) D_{k^{\prime}, m^{\prime}}^{\left(l^{\prime}\right)}(\Omega) D_{k^{\prime \prime}, m^{\prime \prime}}^{\left(l^{\prime \prime}\right)}(\Omega) \\
& \times D_{k^{\prime \prime \prime}, m^{\prime \prime \prime}}^{\left(l^{\prime \prime \prime}\right)}(\Omega) d \Omega
\end{aligned}
$$

and evaluating the integral we find

$$
\begin{aligned}
\int D_{k, m}^{(l)}(\Omega) D_{k^{\prime}, m^{\prime}}^{\left(l^{\prime}\right)}(\Omega) D_{k^{\prime \prime}, m^{\prime \prime}}^{\left(l^{\prime \prime}\right)}(\Omega) D_{k^{\prime \prime \prime}, m^{\prime \prime \prime}}^{\left(l^{\prime \prime \prime}\right)}(\Omega) d \Omega \\
=8 \pi^{2} \sum_{p, q, r, s} F_{l k m p} F_{l^{\prime} k^{\prime} m^{\prime} q^{\prime}} F_{l^{\prime \prime} k^{\prime \prime} m^{\prime \prime} r} F_{l^{\prime \prime \prime} k^{\prime \prime \prime} m^{\prime \prime \prime} s} \\
\quad \times \delta_{\left(m+m^{\prime}+m^{\prime \prime}+m^{\prime \prime \prime}\right) 0} \delta_{\left(k+k^{\prime}+k^{\prime \prime}+k^{\prime \prime \prime}\right) 0} \\
\quad \times B\left(1+p+q+r+s, 1+l+l^{\prime}\right. \\
\left.\quad+l^{\prime \prime}+l^{\prime \prime \prime}-p-q-r-s\right),
\end{aligned}
$$


where $B(x, y)$ is the $\beta$ function [25] and we have defined

$$
F_{l k m p}=(-1)^{p} \frac{\sqrt{(l+m) !(l-m) !(l+k) !(l-k) !}}{p !(l-k-p) !(l+m-p) !(k-m+p) !} .
$$

Using Eqs. (58), (59), and (64) and the properties of the $\beta$ function we obtain

$$
\begin{aligned}
F_{(\lambda \tilde{\delta}, \mu \tilde{\varepsilon}, \nu \tilde{\phi})}^{(l ; k, m)} & \frac{8 \pi^{2}}{(l+4) !} \sum_{k^{\prime}, m^{\prime}} \sum_{k^{\prime \prime}, m^{\prime \prime}} \sum_{p, \ldots, s} a_{\lambda \tilde{\delta}}^{\left(1, k^{\prime}, m^{\prime}\right)} a_{\mu \tilde{\varepsilon}}^{\left(1, k^{\prime \prime}, m^{\prime \prime}\right)} \\
& \times a_{\nu \tilde{\phi}}^{\left(1,-k-k^{\prime}-k^{\prime \prime},-m-m^{\prime}-m^{\prime \prime}\right)} F_{l k m p} F_{1 k^{\prime} m^{\prime} q} \\
& \times F_{1 k^{\prime \prime} m^{\prime \prime} r} F_{1\left(-k-k^{\prime}-k^{\prime \prime}\right)\left(-m-m^{\prime}-m^{\prime \prime}\right) s} \\
& \times(p+q+r+s) !(l+3-p-q-r-s) !
\end{aligned}
$$

Finally, using the expression for $a_{\lambda \tilde{\delta}}^{(l, k, m)}$ given in Eqs. (58) and (59), we have

$$
\begin{aligned}
F_{(\lambda \tilde{\delta}, \mu \tilde{\varepsilon}, \nu \tilde{\phi})}^{(l ; k, m)}= & \frac{\pi^{2}}{(l+4) !} \sum_{t, \ldots, w=1}^{3} \Delta_{t u}^{(\lambda \tilde{\delta})} \Delta_{\nu w}^{(\mu \tilde{\varepsilon})} \\
& \times \Delta_{(6+k-t-\nu)(6+m-u-w)}^{(\nu \tilde{\phi})} \\
& \times \sum_{p, \ldots, s} F_{l k m p} F_{1(2-t)(2-u) q} F_{1(2-\nu)(2-w) r} \\
& \times F_{1(t+\nu-k-4)(u+w-m-4) s}(p+q+r+s) ! \\
& \times(l+3-p-q-r-s) ! .
\end{aligned}
$$

This equation may be used to generate expressions for $F_{(\lambda \tilde{\delta}, \mu \tilde{\epsilon}, \nu \tilde{\phi})}^{(l ; k, m)}$. When $l, k$, and $m$ are set equal to zero we arrive at the rather cumbersome expression:

$$
\begin{aligned}
& F_{(\lambda \tilde{\delta}, \mu \tilde{\varepsilon}, \nu \tilde{\phi})}^{(0 ; 0,0)}=\frac{\pi^{2}}{6}\left\{\Delta_{11}^{(\lambda \tilde{\delta})} \Delta_{22}^{(\mu \tilde{\varepsilon})} \Delta_{33}^{(\nu \tilde{\phi})}-\Delta_{11}^{(\lambda \tilde{\delta})} \Delta_{23}^{(\mu \widetilde{\varepsilon})} \Delta_{32}^{(\nu \tilde{\phi})}-\Delta_{11}^{(\lambda \tilde{\delta})} \Delta_{32}^{(\mu \tilde{\varepsilon})} \Delta_{23}^{(\nu \tilde{\phi})}+\Delta_{11}^{(\lambda \tilde{\delta})} \Delta_{33}^{(\mu \tilde{\varepsilon})} \Delta_{22}^{(\nu \tilde{\phi})}\right. \\
& -\Delta_{12}^{(\lambda \tilde{\delta})} \Delta_{21}^{(\mu \widetilde{\varepsilon})} \Delta_{33}^{(\nu \tilde{\phi})}+\Delta_{12}^{(\lambda \tilde{\delta})} \Delta_{23}^{(\mu \widetilde{\varepsilon})} \Delta_{31}^{(\nu \tilde{\phi})}+\Delta_{12}^{(\lambda \tilde{\delta})} \Delta_{31}^{(\mu \widetilde{\varepsilon})} \Delta_{23}^{(\nu \tilde{\phi})}-\Delta_{12}^{(\lambda \tilde{\delta})} \Delta_{33}^{(\mu \widetilde{\varepsilon})} \Delta_{21}^{(\nu \tilde{\phi})} \\
& +\Delta_{13}^{(\lambda \tilde{\delta})} \Delta_{21}^{(\mu \tilde{\varepsilon})} \Delta_{32}^{(\nu \tilde{\phi})}-\Delta_{13}^{(\lambda \tilde{\delta})} \Delta_{22}^{(\mu \tilde{\varepsilon})} \Delta_{31}^{(\nu \tilde{\phi})}-\Delta_{13}^{(\lambda \tilde{\delta})} \Delta_{31}^{(\mu \tilde{\varepsilon})} \Delta_{22}^{(\nu \tilde{\phi})}+\Delta_{13}^{(\lambda \tilde{\delta})} \Delta_{32}^{(\mu \tilde{\varepsilon})} \Delta_{21}^{(\nu \tilde{\phi})} \\
& -\Delta_{21}^{(\lambda \tilde{\delta})} \Delta_{12}^{(\mu \tilde{\varepsilon})} \Delta_{33}^{(\nu \tilde{\phi})}+\Delta_{21}^{(\lambda \tilde{\delta})} \Delta_{13}^{(\mu \tilde{\varepsilon})} \Delta_{32}^{(\nu \tilde{\phi})}+\Delta_{21}^{(\lambda \tilde{\delta})} \Delta_{32}^{(\mu \tilde{\varepsilon})} \Delta_{13}^{(\nu \tilde{\phi})}-\Delta_{21}^{(\lambda \tilde{\delta})} \Delta_{33}^{(\mu \tilde{\varepsilon})} \Delta_{12}^{(\nu \tilde{\phi})} \\
& +\Delta_{22}^{(\lambda \tilde{\delta})} \Delta_{11}^{(\mu \widetilde{\varepsilon})} \Delta_{33}^{(\nu \tilde{\phi})}-\Delta_{22}^{(\lambda \tilde{\delta})} \Delta_{13}^{(\mu \tilde{\varepsilon})} \Delta_{31}^{(\nu \tilde{\phi})}-\Delta_{22}^{(\lambda \tilde{\delta})} \Delta_{31}^{(\mu \widetilde{\varepsilon})} \Delta_{13}^{(\nu \tilde{\phi})}+\Delta_{22}^{(\lambda \tilde{\delta})} \Delta_{33}^{(\mu \widetilde{\varepsilon})} \Delta_{11}^{(\nu \tilde{\phi})}-\Delta_{23}^{(\lambda \tilde{\delta})} \Delta_{11}^{(\mu \widetilde{\varepsilon})} \Delta_{32}^{(\nu \tilde{\phi})} \\
& +\Delta_{23}^{(\lambda \tilde{\delta})} \Delta_{12}^{(\mu \widetilde{\varepsilon})} \Delta_{31}^{(\nu \tilde{\phi})}+\Delta_{23}^{(\lambda \tilde{\delta})} \Delta_{31}^{(\mu \tilde{\varepsilon})} \Delta_{12}^{(\nu \tilde{\phi})}-\Delta_{23}^{(\lambda \tilde{\delta})} \Delta_{32}^{(\mu \widetilde{\varepsilon})} \Delta_{11}^{(\nu \tilde{\phi})}+\Delta_{31}^{(\lambda \tilde{\delta})} \Delta_{12}^{(\mu \widetilde{\varepsilon})} \Delta_{23}^{(\nu \tilde{\phi})}-\Delta_{31}^{(\lambda \tilde{\delta})} \Delta_{13}^{(\mu \widetilde{\varepsilon})} \Delta_{22}^{(\nu \tilde{\phi})} \\
& -\Delta_{31}^{(\lambda \tilde{\delta})} \Delta_{22}^{(\mu \tilde{\varepsilon})} \Delta_{13}^{(\nu \widetilde{\phi})}+\Delta_{31}^{(\lambda \tilde{\delta})} \Delta_{23}^{(\mu \tilde{\varepsilon})} \Delta_{12}^{(\nu \tilde{\phi})}-\Delta_{32}^{(\lambda \tilde{\delta})} \Delta_{11}^{(\mu \widetilde{\varepsilon})} \Delta_{23}^{(\nu \tilde{\phi})}+\Delta_{32}^{(\lambda \tilde{\delta})} \Delta_{13}^{(\mu \widetilde{\varepsilon})} \Delta_{21}^{(\nu \tilde{\phi})}+\Delta_{32}^{(\lambda \tilde{\delta})} \Delta_{21}^{(\mu \tilde{\varepsilon})} \Delta_{13}^{(\nu \tilde{\phi})} \\
& \left.-\Delta_{32}^{(\lambda \tilde{\delta})} \Delta_{23}^{(\mu \tilde{\varepsilon})} \Delta_{11}^{(\nu \tilde{\phi})}+\Delta_{33}^{(\lambda \tilde{\delta})} \Delta_{11}^{(\mu \tilde{\varepsilon})} \Delta_{22}^{(\nu \tilde{\phi})}-\Delta_{33}^{(\lambda \tilde{\delta})} \Delta_{12}^{(\mu \tilde{\varepsilon})} \Delta_{21}^{(\nu \tilde{\phi})}-\Delta_{33}^{(\lambda \tilde{\delta})} \Delta_{21}^{(\mu \tilde{\varepsilon})} \Delta_{12}^{(\nu \tilde{\phi})}+\Delta_{33}^{(\lambda \tilde{\delta})} \Delta_{22}^{(\mu \tilde{\varepsilon})} \Delta_{11}^{(\nu \tilde{\phi})}\right\} .
\end{aligned}
$$

However, $D_{0,0}^{(0)}(\Omega)=1$ and so $F_{(\lambda \widetilde{\delta}, \mu \widetilde{\varepsilon}, \nu \tilde{\phi})}^{(0 ; 0,0)}$ is related to the isotropic average of a product of three direction cosines-the result of which is known [26]. Overall one finds

$$
F_{(\lambda \tilde{\delta}, \mu \tilde{\varepsilon}, \nu \tilde{\phi})}^{(0: 0,0)}=\frac{4 \pi^{2}}{3} \varepsilon_{\lambda \mu \nu} \varepsilon \tilde{\delta} \tilde{\varepsilon} \tilde{\phi} .
$$

The equality between Eqs. (69) and (70) may be used to generate relations between the $\delta$ 's. For example, setting $\lambda$ $=x$ and $\widetilde{\delta}=x$, we see from Eq. (59) that only four terms are nonzero, namely, $\Delta_{11}^{(x x)}=1, \Delta_{13}^{(x x)}=-1, \Delta_{31}^{(x x)}=-1$, and $\Delta_{33}^{(x x)}=1$. Inserting these into Eq. (69) we get 


$$
\begin{aligned}
& \left\{\Delta_{22}^{(\mu \widetilde{\varepsilon})} \Delta_{33}^{(\nu \tilde{\phi})}-\Delta_{23}^{(\mu \widetilde{\varepsilon})} \Delta_{32}^{(\nu \tilde{\phi})}-\Delta_{32}^{(\mu \tilde{\varepsilon})} \Delta_{23}^{(\nu \widetilde{\phi})}+\Delta_{33}^{(\mu \widetilde{\varepsilon})} \Delta_{22}^{(\nu \widetilde{\phi})}\right. \\
& -\Delta_{21}^{(\mu \tilde{\varepsilon})} \Delta_{32}^{(\nu \tilde{\phi})}+\Delta_{22}^{(\mu \tilde{\varepsilon})} \Delta_{31}^{(\nu \tilde{\phi})}+\Delta_{31}^{(\mu \tilde{\varepsilon})} \Delta_{22}^{(\nu \tilde{\phi})}-\Delta_{32}^{(\mu \widetilde{\varepsilon})} \Delta_{21}^{(\nu \tilde{\phi})} \\
& -\Delta_{12}^{(\mu \widetilde{\varepsilon})} \Delta_{23}^{(\nu \widetilde{\phi})}+\Delta_{13}^{(\mu \widetilde{\varepsilon})} \Delta_{22}^{(\nu \widetilde{\phi})}+\Delta_{22}^{(\mu \widetilde{\varepsilon})} \Delta_{13}^{(\nu \widetilde{\phi})}-\Delta_{23}^{(\mu \widetilde{\varepsilon})} \Delta_{12}^{(\nu \widetilde{\phi})} \\
& +\Delta_{11}^{(\mu \widetilde{\varepsilon})} \Delta_{22}^{(\nu \tilde{\phi})}-\Delta_{12}^{(\mu \tilde{\varepsilon})} \Delta_{21}^{(\nu \tilde{\phi})}-\Delta_{21}^{(\mu \widetilde{\varepsilon})} \Delta_{12}^{(\nu \tilde{\phi})} \\
& \left.+\Delta_{22}^{(\mu \widetilde{\varepsilon})} \Delta_{11}^{(\nu \tilde{\phi})}\right\} \\
& =8 \varepsilon_{x \mu \nu} \varepsilon_{x \tilde{\varepsilon} \tilde{\phi}} .
\end{aligned}
$$

In this way we can generate general expressions that must hold for sums of products of two $\delta$ 's. In particular it follows that

$$
\begin{aligned}
\left\{\Delta_{22}^{(\mu \widetilde{\varepsilon})}\right. & \Delta_{33}^{(\nu \tilde{\phi})}-\Delta_{23}^{(\mu \widetilde{\varepsilon})} \Delta_{32}^{(\nu \tilde{\phi})}-\Delta_{32}^{(\mu \widetilde{\varepsilon})} \Delta_{23}^{(\nu \tilde{\phi})}+\Delta_{33}^{(\mu \widetilde{\varepsilon})} \Delta_{22}^{(\nu \tilde{\phi})} \\
& +\Delta_{21}^{(\mu \widetilde{\varepsilon})} \Delta_{32}^{(\nu \widetilde{\phi})}-\Delta_{22}^{(\mu \widetilde{\varepsilon})} \Delta_{31}^{(\nu \widetilde{\phi})}-\Delta_{31}^{(\mu \widetilde{\varepsilon})} \Delta_{22}^{(\nu \widetilde{\phi})}+\Delta_{32}^{(\mu \widetilde{\varepsilon})} \Delta_{21}^{(\nu \widetilde{\phi})} \\
& +\Delta_{12}^{(\mu \widetilde{\varepsilon})} \Delta_{23}^{(\nu \widetilde{\phi})}-\Delta_{13}^{(\mu \widetilde{\varepsilon})} \Delta_{22}^{(\nu \widetilde{\phi})}-\Delta_{22}^{(\mu \widetilde{\varepsilon})} \Delta_{13}^{(\nu \widetilde{\phi})}+\Delta_{23}^{(\mu \widetilde{\varepsilon})} \Delta_{12}^{(\nu \widetilde{\phi})} \\
& \left.+\Delta_{11}^{(\mu \widetilde{\varepsilon})} \Delta_{22}^{(\nu \tilde{\phi})}-\Delta_{12}^{(\mu \widetilde{\varepsilon})} \Delta_{21}^{(\nu \tilde{\phi})}-\Delta_{21}^{(\mu \widetilde{\varepsilon})} \Delta_{12}^{(\nu \widetilde{\phi})}+\Delta_{22}^{(\mu \widetilde{\varepsilon})} \Delta_{11}^{(\nu \widetilde{\phi})}\right\} \\
= & 8 \varepsilon_{y \mu \nu} \varepsilon_{y \widetilde{\varepsilon} \tilde{\phi}}
\end{aligned}
$$

and so we may use Eq. (71) and Eq. (72) to write

$$
\begin{aligned}
\left\{\Delta_{22}^{(\mu \widetilde{\varepsilon})}\right. & \Delta_{33}^{(\nu \tilde{\phi})}-\Delta_{23}^{(\mu \widetilde{\varepsilon})} \Delta_{32}^{(\nu \widetilde{\phi})}-\Delta_{32}^{(\mu \tilde{\varepsilon})} \Delta_{23}^{(\nu \widetilde{\phi})} \\
& +\Delta_{33}^{(\mu \tilde{\varepsilon})} \Delta_{22}^{(\nu \widetilde{\phi})}+\Delta_{11}^{(\mu \widetilde{\varepsilon})} \Delta_{22}^{(\nu \widetilde{\phi})}-\Delta_{12}^{(\mu \widetilde{\varepsilon})} \Delta_{21}^{(\nu \tilde{\phi})} \\
& \left.-\Delta_{21}^{(\mu \tilde{\varepsilon})} \Delta_{12}^{(\nu \widetilde{\phi})}+\Delta_{22}^{(\mu \widetilde{\varepsilon})} \Delta_{11}^{(\nu \tilde{\phi})}\right\} \\
= & 4\left(\varepsilon_{x \mu \nu} \varepsilon_{x \tilde{\varepsilon} \tilde{\phi}}+\varepsilon_{y \mu \nu} \varepsilon_{y \tilde{\varepsilon} \tilde{\phi}}\right)
\end{aligned}
$$

and

$$
\begin{aligned}
\left\{\Delta_{21}^{(\mu \tilde{\varepsilon})}\right. & \Delta_{32}^{(\nu \tilde{\phi})}-\Delta_{22}^{(\mu \widetilde{\varepsilon})} \Delta_{31}^{(\nu \widetilde{\phi})}-\Delta_{31}^{(\mu \widetilde{\varepsilon})} \Delta_{22}^{(\nu \widetilde{\phi})}+\Delta_{32}^{(\mu \widetilde{\varepsilon})} \Delta_{21}^{(\nu \tilde{\phi})} \\
& \left.+\Delta_{12}^{(\mu \widetilde{\varepsilon})} \Delta_{23}^{(\nu \tilde{\phi})}-\Delta_{13}^{(\mu \widetilde{\varepsilon})} \Delta_{22}^{(\nu \tilde{\phi})}-\Delta_{22}^{(\mu \widetilde{\varepsilon})} \Delta_{13}^{(\nu \widetilde{\phi})}+\Delta_{23}^{(\mu \widetilde{\varepsilon})} \Delta_{12}^{(\nu \widetilde{\phi})}\right\} \\
= & -4\left(\varepsilon_{x \mu \nu} \varepsilon_{x \tilde{\varepsilon} \tilde{\phi}}-\varepsilon_{y \mu \nu} \varepsilon_{y \tilde{\varepsilon} \tilde{\phi}}\right) .
\end{aligned}
$$

This reduction process is aided by use of the properties of the $\Delta$ matrices given in Eqs. (60)-(63). The equations thus produced can be checked directly, confirming our assertion that Eqs. (69) and (70) are equivalent. They may also be used to simplify the other expressions generated by Eq. (68). The overall results for $G_{(\lambda \tilde{\delta}, \mu \tilde{\varepsilon}, \nu \tilde{\phi})}^{(l ; \eta)}$ are shown in Table II. Using Tables I and II and Eqs. (35), (36), (49) we can deduce the temporal variation of the six-wave mixing signal for any choice of beam polarizations. We shall do this now for some cases of interest.

\section{TEMPORAL PROFILES}

From now on we assume that the lifetime of the excited state is much longer than the timescale of rotational reorien- tation. From Eq. (35) we see that the temporal variation of the harmonic signal may be written

$$
I_{\mathrm{sig}}(\tau)=\kappa\left|\sum_{n=1}^{6} \Lambda_{n}(\tau) E_{n}\right|^{2}
$$

where the time dependence is shown explicitly so that $\kappa$ is a time-independent constant, and the $E_{n}$ are scalar parameters determined by the polarization conditions, as given by Eq. (26). We further note that Eq. (36) may be rewritten in the form

$$
\Lambda(\tau)=\mathbf{M}\left[\begin{array}{c}
\bar{\mu}_{\lambda}^{10} \alpha_{(\mu \nu)}^{10} \Delta \beta_{\tilde{\alpha}(\tilde{\gamma} \tilde{\gamma})} F_{(\lambda \tilde{\alpha}, \mu \tilde{\beta}, \nu \tilde{\beta})} \\
\bar{\mu}_{\mu}^{10} \alpha_{(\lambda \nu)}^{10} \Delta \beta_{\tilde{\alpha}(\tilde{\gamma} \tilde{)})} F_{(\lambda \tilde{\alpha}, \mu \tilde{\beta}, \nu \tilde{\beta})} \\
\bar{\mu}_{\lambda}^{10} \alpha_{(\mu \nu)}^{10} \Delta \beta_{\tilde{\alpha}(\tilde{\beta} \tilde{\gamma})} F_{(\lambda \tilde{\alpha}, \mu \tilde{\beta}, \nu \tilde{\gamma})} \\
\bar{\mu}_{\lambda}^{10} \alpha_{(\mu \nu)}^{10} \Delta \beta_{\tilde{\beta}(\tilde{\gamma} \tilde{\alpha})} F_{(\lambda \tilde{\alpha}, \mu \tilde{\beta}, \nu \tilde{\gamma})} \\
\bar{\mu}_{\mu}^{10} \alpha_{(\lambda \nu)}^{10} \Delta \beta_{\tilde{\gamma}(\tilde{\alpha} \tilde{\gamma})} F_{(\lambda \tilde{\alpha}, \mu \tilde{\beta}, \nu \tilde{\beta})} \\
\bar{\mu}_{\lambda}^{10} \alpha_{(\mu \nu)}^{10} \Delta \beta_{\tilde{\gamma}(\tilde{\alpha} \tilde{\gamma})} F_{(\lambda \tilde{\alpha}, \mu \tilde{\beta}, \nu \tilde{\beta})}
\end{array}\right],
$$

and since the time-dependence here lies within the $\mathbf{F}$ tensor we see that there are in fact two different ways in which time features: through a fourth-rank contracted (single pair trace) tensor of the form $F_{(\lambda \tilde{\alpha}, \mu \tilde{\beta}, \nu \widetilde{\beta})}$ and through the sixth-rank tensor $F_{(\lambda \tilde{\alpha}, \mu \tilde{\beta}, \nu \tilde{\gamma})}$. Tables I and II easily allow us to find the form for the contracted tensor-we find that it is only when $l=1$ that nonzero contributions arise, i.e., the only terms to survive involve

$$
G_{(\lambda \widetilde{\delta}, \mu \tilde{\varepsilon}, \nu \widetilde{\varepsilon})}^{(1 ; \eta)}= \begin{cases}\delta_{\tilde{\delta} y} \delta_{\lambda y} \delta_{\mu \nu}, & \eta=1 \\ \delta_{\tilde{\delta} z} \delta_{\lambda z} \delta_{\mu \nu}, & \eta=0 \\ \delta_{\tilde{\delta} x} \delta_{\lambda x} \delta_{\mu \nu}, & \eta=-1,\end{cases}
$$

so that

$$
\begin{aligned}
F_{(\lambda \tilde{\alpha}, \mu \tilde{\beta}, \nu \tilde{\beta})}= & \delta_{\mu \nu}\left\{\delta_{\tilde{\alpha} y} \delta_{\lambda y} e^{-E_{1}^{(1)} \tau}+\delta_{\tilde{\alpha} z} \delta_{\lambda z} e^{-E_{0}^{(1)} \tau}\right. \\
& \left.+\delta_{\tilde{\alpha} x} \delta_{\lambda x} e^{-E_{-1}^{(1)} \tau}\right\} .
\end{aligned}
$$

Quite generally then, the temporal evolution of the signal may be written as

$$
I_{\mathrm{sig}}(\tau)=\kappa\left|\chi_{\lambda \mu \nu \tilde{\alpha}}^{(3)} F_{(\lambda \tilde{\alpha}, \mu \tilde{\beta}, \nu \tilde{\beta})}+\chi_{\lambda(\mu \nu) \tilde{\alpha} \tilde{\beta} \tilde{\gamma}}^{(5)} F_{(\lambda \tilde{\alpha}, \mu \tilde{\beta}, \nu \tilde{\gamma})}\right|^{2},
$$

where

$$
\begin{aligned}
\chi_{\lambda \mu \nu \tilde{\alpha}}^{(3)}= & \frac{1}{105}\left\{\overline { \mu } _ { \lambda } ^ { 1 0 } \alpha _ { ( \mu \nu ) } ^ { 1 0 } \Delta \beta _ { \tilde { \alpha } ( \tilde { \gamma } \tilde { \gamma } ) } \left(8 E_{1}-5 E_{2}-5 E_{3}+4 E_{4}\right.\right. \\
& \left.+4 E_{5}-5 E_{6}\right)+\bar{\mu}_{\mu}^{10} \alpha_{(\lambda \nu)}^{10} \Delta \beta_{\tilde{\alpha}(\tilde{\gamma} \tilde{\gamma})} \\
& \times\left(-5 E_{1}+11 E_{2}+4 E_{3}-6 E_{4}-6 E_{5}+4 E_{6}\right) \\
& +\bar{\mu}_{\mu}^{10} \alpha_{(\lambda \nu)}^{10} \Delta \beta_{\tilde{\gamma}(\tilde{\alpha} \tilde{\gamma})}\left(4 E_{1}-6 E_{2}-6 E_{3}+2 E_{4}\right. \\
& \left.+16 E_{5}-6 E_{6}\right)+\bar{\mu}_{\lambda}^{10} \alpha_{(\mu \nu)}^{10} \Delta \beta_{\tilde{\gamma}(\tilde{\alpha} \tilde{\gamma})} \\
& \left.\times\left(-5 E_{1}+4 E_{2}+4 E_{3}-6 E_{4}-6 E_{5}+11 E_{6}\right)\right\}
\end{aligned}
$$


TABLE II. Explicit forms of the $G_{(\lambda \tilde{\delta}, \mu \tilde{\varepsilon}, \nu \tilde{\phi})}^{(l ; \eta)}$ coefficients for $l \leqslant 3$.

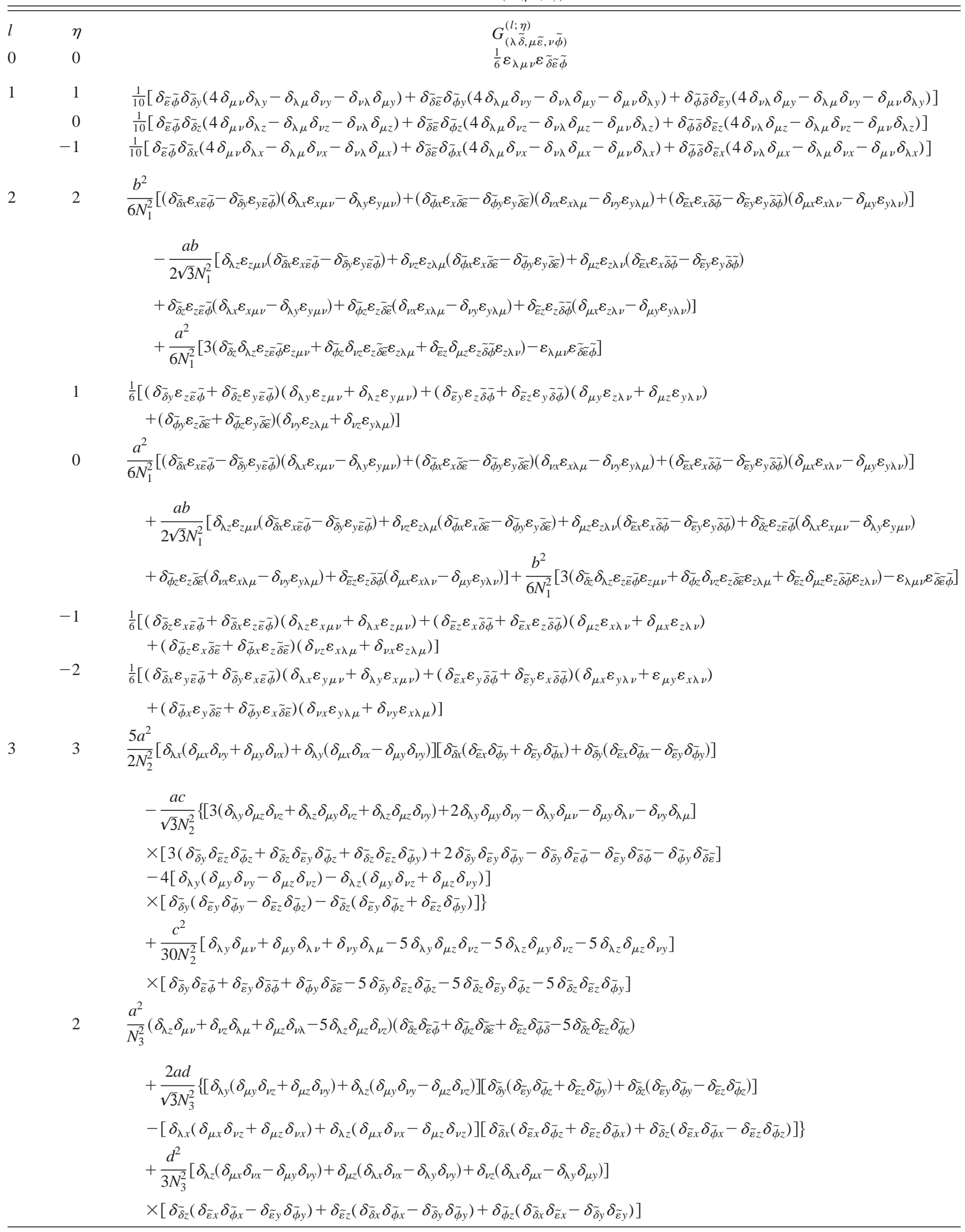


TABLE II. (Continued).

$$
\begin{aligned}
& 1 \quad \frac{c^{2}}{2 N_{2}^{2}}\left[\delta_{\lambda x}\left(\delta_{\mu x} \delta_{\nu y}+\delta_{\mu y} \delta_{\nu x}\right)+\delta_{\lambda y}\left(\delta_{\mu x} \delta_{\nu x}-\delta_{\mu y} \delta_{\nu y}\right)\right]\left[\delta_{\tilde{\delta x}}\left(\delta_{\tilde{\delta} x} \delta_{\tilde{\phi} y}+\delta_{\tilde{\varepsilon} y} \delta_{\tilde{\phi} x}\right)+\delta_{\tilde{\delta y}}\left(\delta_{\tilde{\varepsilon} x} \delta_{\tilde{\phi} x}-\delta_{\tilde{y}} \delta_{\tilde{\phi} y}\right)\right] \\
& +\frac{a c}{\sqrt{3} N_{2}^{2}}\left\{\left[3\left(\delta_{\lambda y} \delta_{\mu z} \delta_{\nu z}+\delta_{\lambda z} \delta_{\mu y} \delta_{\nu z}+\delta_{\lambda z} \delta_{\mu z} \delta_{\nu y}\right)+2 \delta_{\lambda y} \delta_{\mu y} \delta_{\nu y}-\delta_{\lambda y} \delta_{\mu \nu}-\delta_{\mu y} \delta_{\lambda \nu}-\delta_{\nu y} \delta_{\lambda \mu}\right]\right. \\
& \times\left[3\left(\delta_{\tilde{\delta} y} \delta_{\tilde{\varepsilon} z} \delta_{\tilde{\phi} z}+\delta_{\tilde{\delta} z} \delta_{\tilde{\varepsilon} y} \delta_{\tilde{\phi} z}+\delta_{\tilde{\delta} z} \delta_{\tilde{\varepsilon} z} \delta_{\tilde{\phi} y}\right)+2 \delta_{\tilde{\delta} y} \delta_{\tilde{\varepsilon} y} \delta_{\tilde{\phi} y}-\delta_{\tilde{\delta} y} \delta_{\tilde{\varepsilon} \tilde{\phi}}-\delta_{\tilde{\varepsilon} y} \delta_{\tilde{\delta} \tilde{\phi}}-\delta_{\tilde{\phi} y} \delta_{\tilde{\delta} \tilde{\varepsilon}}\right] \\
& \left.-4\left[\delta_{\lambda y}\left(\delta_{\mu y} \delta_{\nu y}-\delta_{\mu z} \delta_{\nu z}\right)-\delta_{\lambda z}\left(\delta_{\mu y} \delta_{\nu z}+\delta_{\mu z} \delta_{\nu y}\right)\right]\left[\delta_{\tilde{\delta} y}\left(\delta_{\tilde{\varepsilon} y} \delta_{\tilde{\phi} y}-\delta_{\tilde{\varepsilon} z} \delta_{\tilde{\phi} z}\right)-\delta_{\tilde{\delta} z}\left(\delta_{\tilde{\varepsilon} y} \delta_{\tilde{\phi} z}+\delta_{\tilde{\varepsilon} z} \delta_{\tilde{\phi} y}\right)\right]\right\} \\
& +\frac{a^{2}}{6 N_{2}^{2}}\left[\delta_{\lambda y} \delta_{\mu \nu}+\delta_{\mu y} \delta_{\lambda \nu}+\delta_{\nu y} \delta_{\lambda \mu}-5 \delta_{\lambda y} \delta_{\mu z} \delta_{\nu z}-5 \delta_{\lambda z} \delta_{\mu y} \delta_{\nu z}-5 \delta_{\lambda z} \delta_{\mu z} \delta_{\nu y}\right] \\
& \times\left[\delta_{\tilde{\delta} y} \delta_{\tilde{\varepsilon} \tilde{\phi}}+\delta_{\tilde{\varepsilon} y} \delta_{\tilde{\delta} \tilde{\phi}}+\delta_{\tilde{\phi} y} \delta_{\tilde{\delta} \tilde{\varepsilon}}-5 \delta_{\tilde{\delta} y} \delta_{\tilde{\varepsilon} z} \delta_{\tilde{\phi} z}-5 \delta_{\tilde{\delta} z} \delta_{\tilde{\varepsilon} y} \delta_{\tilde{\phi} z}-5 \delta_{\tilde{\delta} z} \delta_{\tilde{\varepsilon} z} \delta_{\tilde{\phi} y}\right] \\
& 0 \quad \frac{a^{2}}{15 N_{3}^{2}}\left[\delta_{\lambda z}\left(\delta_{\mu x} \delta_{\nu x}-\delta_{\mu y} \delta_{\nu y}\right)+\delta_{\mu z}\left(\delta_{\lambda x} \delta_{\nu x}-\delta_{\lambda y} \delta_{\nu y}\right)+\delta_{\nu z}\left(\delta_{\lambda x} \delta_{\mu x}-\delta_{\lambda y} \delta_{\mu y}\right)\right] \\
& \times\left[\delta_{\tilde{\delta} z}\left(\delta_{\tilde{\varepsilon} x} \delta_{\tilde{\phi} x}-\delta_{\tilde{\varepsilon} y} \delta_{\tilde{\phi} y}\right)+\delta_{\tilde{\varepsilon} z}\left(\delta_{\tilde{\delta} x} \delta_{\tilde{\phi} x}-\delta_{\tilde{\delta} y} \delta_{\tilde{\phi} y}\right)+\delta_{\tilde{\phi} z}\left(\delta_{\tilde{\delta} x} \delta_{\tilde{\varepsilon} x}-\delta_{\tilde{\delta} y} \delta_{\tilde{\varepsilon} y}\right)\right] \\
& -\frac{2 a d}{5 \sqrt{3} N_{3}^{2}}\left\{\left[\delta_{\lambda y}\left(\delta_{\mu y} \delta_{v z}+\delta_{\mu z} \delta_{\nu y}\right)+\delta_{\lambda z}\left(\delta_{\mu y} \delta_{\nu y}-\delta_{\mu z} \delta_{v z}\right)\right]\left[\delta_{\tilde{\delta y}}\left(\delta_{\tilde{\varepsilon} y} \delta_{\tilde{\phi} z}+\delta_{\tilde{\varepsilon} z} \delta_{\tilde{\phi y}}\right)+\delta_{\tilde{\delta} z}\left(\delta_{\tilde{\varepsilon} y} \delta_{\tilde{\phi y}}-\delta_{\tilde{\varepsilon} z} \delta_{\tilde{\phi} z}\right)\right]\right. \\
& \left.-\left[\delta_{\lambda x}\left(\delta_{\mu x} \delta_{\nu z}+\delta_{\mu z} \delta_{\nu x}\right)+\delta_{\lambda z}\left(\delta_{\mu x} \delta_{\nu x}-\delta_{\mu z} \delta_{\nu z}\right)\right]\left[\delta_{\tilde{\delta} x}\left(\delta_{\tilde{\varepsilon} x} \delta_{\tilde{\phi} z}+\delta_{\tilde{\varepsilon} z} \delta_{\tilde{\phi} x}\right)+\delta_{\tilde{\delta} z}\left(\delta_{\tilde{\varepsilon} x} \delta_{\tilde{\phi} x}-\delta_{\tilde{\varepsilon} z} \delta_{\tilde{\phi} z}\right)\right]\right\} \\
& +\frac{d^{2}}{5 N_{3}^{2}}\left(\delta_{\lambda z} \delta_{\mu \nu}+\delta_{\nu z} \delta_{\lambda \mu}+\delta_{\mu z} \delta_{\nu \lambda}-5 \delta_{\lambda z} \delta_{\mu z} \delta_{\nu z}\right)\left(\delta_{\tilde{\delta} z} \delta_{\tilde{\delta} \tilde{\phi}}+\delta_{\tilde{\phi} z} \delta_{\tilde{\delta} \tilde{\varepsilon}}+\delta_{\tilde{\varepsilon} z} \delta_{\tilde{\phi} \tilde{\delta}}-5 \delta_{\tilde{\delta} z} \delta_{\tilde{\varepsilon} z} \delta_{\tilde{\phi} z}\right) \\
& -1 \quad \frac{a^{2}}{10 N_{4}^{2}}\left[\delta_{\lambda x}\left(\delta_{\mu x} \delta_{\nu x}-\delta_{\mu y} \delta_{\nu y}\right)-\delta_{\lambda y}\left(\delta_{\mu y} \delta_{\nu x}+\delta_{\mu x} \delta_{\nu y}\right)\right]\left[\delta_{\tilde{\delta} x}\left(\delta_{\tilde{\varepsilon} x} \delta_{\tilde{\phi} x}-\delta_{\tilde{\varepsilon} y} \delta_{\tilde{\phi} y}\right)-\delta_{\tilde{\delta} y}\left(\delta_{\tilde{\varepsilon} x} \delta_{\tilde{\phi} y}+\delta_{\tilde{\varepsilon} y} \delta_{\tilde{\phi} x}\right)\right] \\
& +\frac{a e}{5 \sqrt{3} N_{4}^{2}}\left\{\left[3\left(\delta_{\lambda x} \delta_{\mu z} \delta_{\nu z}+\delta_{\lambda z} \delta_{\mu x} \delta_{\nu z}+\delta_{\lambda z} \delta_{\mu z} \delta_{\nu x}\right)+2 \delta_{\lambda x} \delta_{\mu x} \delta_{\nu x}-\delta_{\lambda x} \delta_{\mu \nu}-\delta_{\mu x} \delta_{\lambda \nu}-\delta_{\nu x} \delta_{\lambda \mu}\right]\right. \\
& \times\left[3\left(\delta_{\tilde{\delta} x} \delta_{\tilde{\varepsilon} z} \delta_{\tilde{\phi} z}+\delta_{\tilde{\delta} z} \delta_{\tilde{\varepsilon} x} \delta_{\tilde{\phi} z}+\delta_{\tilde{\delta} z} \delta_{\tilde{\varepsilon} z} \delta_{\tilde{\phi} x}\right)+2 \delta_{\tilde{\delta} x} \delta_{\tilde{\varepsilon} x} \delta_{\tilde{\phi} x}-\delta_{\tilde{\delta} x} \delta_{\tilde{\varepsilon} \tilde{\phi}}-\delta_{\tilde{\varepsilon} x} \delta_{\tilde{\delta} \tilde{\phi}}-\delta_{\tilde{\phi} x} \delta_{\tilde{\delta} \tilde{\varepsilon}}\right] \\
& \left.-4\left[\delta_{\lambda x}\left(\delta_{\mu x} \delta_{\nu x}-\delta_{\mu z} \delta_{\nu z}\right)-\delta_{\lambda z}\left(\delta_{\mu x} \delta_{\nu z}+\delta_{\mu z} \delta_{\nu x}\right)\right]\left[\delta_{\tilde{\delta} x}\left(\delta_{\tilde{\varepsilon} x} \delta_{\tilde{\phi} x}-\delta_{\tilde{\varepsilon} z} \delta_{\tilde{\phi} z}\right)-\delta_{\tilde{\delta} z}\left(\delta_{\tilde{\varepsilon} x} \delta_{\tilde{\phi} z}+\delta_{\tilde{\varepsilon} z} \delta_{\tilde{\phi} x}\right)\right]\right\} \\
& +\frac{e^{2}}{30 N_{4}^{2}}\left[\delta_{\lambda x} \delta_{\mu \nu}+\delta_{\mu x} \delta_{\lambda \nu}+\delta_{\nu x} \delta_{\lambda \mu}-5 \delta_{\lambda x} \delta_{\mu z} \delta_{\nu z}-5 \delta_{\lambda z} \delta_{\mu x} \delta_{\nu z}-5 \delta_{\lambda z} \delta_{\mu z} \delta_{\nu x}\right] \\
& \times\left[\delta_{\tilde{\delta} x} \delta_{\tilde{\varepsilon} \tilde{\phi}}+\delta_{\tilde{\varepsilon} x} \delta_{\tilde{\delta} \tilde{\phi}}+\delta_{\tilde{\phi} x} \delta_{\tilde{\delta} \tilde{\varepsilon}}-5 \delta_{\tilde{\delta} x} \delta_{\tilde{\varepsilon} z} \delta_{\tilde{\phi} z}-5 \delta_{\tilde{\delta} z} \delta_{\tilde{\varepsilon} x} \delta_{\tilde{\phi} z}-5 \delta_{\tilde{\delta} z} \delta_{\tilde{\varepsilon} z} \delta_{\tilde{\phi} x}\right] \\
& -2 \quad \frac{1}{6}\left[\delta_{\lambda x}\left(\delta_{\mu y} \delta_{\nu z}+\delta_{\mu z} \delta_{\nu y}\right)+\delta_{\lambda y}\left(\delta_{\mu z} \delta_{\nu x}+\delta_{\mu x} \delta_{\nu z}\right)+\delta_{\lambda z}\left(\delta_{\mu x} \delta_{\nu y}+\delta_{\mu y} \delta_{\nu x}\right)\right] \\
& \times\left[\delta_{\tilde{\delta} x}\left(\delta_{\tilde{\varepsilon} y} \delta_{\tilde{\phi} z}+\delta_{\tilde{\varepsilon} z} \delta_{\tilde{\phi} y}\right)+\delta_{\tilde{\delta} y}\left(\delta_{\tilde{\varepsilon} z} \delta_{\tilde{\phi} x}+\delta_{\tilde{\varepsilon} x} \delta_{\tilde{\phi} z}\right)+\delta_{\tilde{\delta} z}\left(\delta_{\tilde{\varepsilon} x} \delta_{\tilde{\phi} y}+\delta_{\tilde{\varepsilon} y} \delta_{\tilde{\phi} x}\right)\right] \\
& -3 \quad \frac{e^{2}}{2 N_{4}^{2}}\left[\delta_{\lambda x}\left(\delta_{\mu x} \delta_{\nu x}-\delta_{\mu y} \delta_{\nu y}\right)-\delta_{\lambda y}\left(\delta_{\mu y} \delta_{\nu x}+\delta_{\mu x} \delta_{\nu y}\right)\right]\left[\delta_{\tilde{\delta x}}\left(\delta_{\tilde{\varepsilon} x} \delta_{\tilde{\phi} x}-\delta_{\tilde{\varepsilon} y} \delta_{\tilde{\phi} y}\right)-\delta_{\tilde{\delta y}}\left(\delta_{\tilde{\varepsilon} x} \delta_{\tilde{\phi} y}+\delta_{\tilde{\varepsilon} y} \delta_{\tilde{\phi} x}\right)\right] \\
& +\frac{a e}{5 \sqrt{3} N_{4}^{2}}\left\{\left[3\left(\delta_{\lambda x} \delta_{\mu z} \delta_{\nu z}+\delta_{\lambda z} \delta_{\mu x} \delta_{\nu z}+\delta_{\lambda z} \delta_{\mu z} \delta_{\nu x}\right)+2 \delta_{\lambda x} \delta_{\mu x} \delta_{\nu x}-\delta_{\lambda x} \delta_{\mu \nu}-\delta_{\mu x} \delta_{\lambda \nu}-\delta_{\nu x} \delta_{\lambda \mu}\right]\right. \\
& \times\left[3\left(\delta_{\tilde{\delta} x} \delta_{\tilde{\varepsilon} z} \delta_{\tilde{\phi} z}+\delta_{\tilde{\delta} z} \delta_{\tilde{\varepsilon} x} \delta_{\tilde{\phi} z}+\delta_{\tilde{\delta} z} \delta_{\tilde{\varepsilon} z} \delta_{\tilde{\phi} x}\right)+2 \delta_{\tilde{\delta} x} \delta_{\tilde{\varepsilon} x} \delta_{\tilde{\phi} x}-\delta_{\tilde{\delta} x} \delta_{\tilde{\varepsilon} \tilde{\phi}}-\delta_{\tilde{\varepsilon} x} \delta_{\tilde{\delta} \tilde{\phi}}-\delta_{\tilde{\phi} x} \delta_{\tilde{\phi} \tilde{\varepsilon}}\right] \\
& \left.-4\left[\delta_{\lambda x}\left(\delta_{\mu x} \delta_{\nu x}-\delta_{\mu z} \delta_{\nu z}\right)-\delta_{\lambda z}\left(\delta_{\mu x} \delta_{\nu z}+\delta_{\mu z} \delta_{\nu x}\right)\right]\left[\delta_{\tilde{\delta} x}\left(\delta_{\tilde{\varepsilon} x} \delta_{\tilde{\phi} x}-\delta_{\tilde{\varepsilon} z} \delta_{\tilde{\phi} z}\right)-\delta_{\tilde{\delta} z}\left(\delta_{\tilde{\varepsilon} x} \delta_{\tilde{\phi} z}+\delta_{\tilde{\varepsilon} z} \delta_{\tilde{\phi} x}\right)\right]\right\} \\
& +\frac{a^{2}}{150 N_{4}^{2}}\left[\delta_{\lambda x} \delta_{\mu \nu}+\delta_{\mu x} \delta_{\lambda \nu}+\delta_{\nu x} \delta_{\lambda \mu}-5 \delta_{\lambda x} \delta_{\mu z} \delta_{\nu z}-5 \delta_{\lambda z} \delta_{\mu x} \delta_{\nu z}-5 \delta_{\lambda z} \delta_{\mu z} \delta_{\nu x}\right] \\
& \times\left[\delta_{\tilde{\delta} x} \delta_{\tilde{\varepsilon} \tilde{\phi}}+\delta_{\tilde{\varepsilon} x} \delta_{\tilde{\delta} \tilde{\phi}}+\delta_{\tilde{\phi} x} \delta_{\tilde{\phi} \tilde{\varepsilon}}-5 \delta_{\tilde{\delta} x} \delta_{\tilde{\varepsilon} z} \delta_{\tilde{\phi} z}-5 \delta_{\tilde{\delta} z} \delta_{\tilde{\varepsilon} x} \delta_{\tilde{\phi} z}-5 \delta_{\tilde{\delta} z} \delta_{\tilde{\varepsilon} z} \delta_{\tilde{\phi} x}\right]
\end{aligned}
$$

and

$$
\begin{aligned}
\chi_{\lambda(\mu \nu) \tilde{\alpha} \tilde{\beta} \tilde{\gamma}}^{(5)}= & \frac{\bar{\mu}_{\lambda}^{10} \alpha_{(\mu \nu)}^{10}}{105}\left\{\Delta \beta _ { \tilde { \alpha } ( \tilde { \beta } \tilde { \gamma } ) } \left(-5 E_{1}+4 E_{2}+11 E_{3}\right.\right. \\
& \left.-6 E_{4}-6 E_{5}+4 E_{6}\right)+\Delta \beta_{\tilde{\beta}(\tilde{\gamma} \tilde{\alpha})} \\
& \left.\times\left(4 E_{1}-6 E_{2}-6 E_{3}+16 E_{4}+2 E_{5}-6 E_{6}\right)\right\}
\end{aligned}
$$

In Eq. (81), the $\mu \nu$ interchange symmetry means that on contraction with $F_{(\lambda \tilde{\alpha}, \mu \tilde{\beta}, \nu \tilde{\gamma})}$ only the $\mu \nu$ symmetric part of that tensor will feature. Thus, on contraction with the purely $\mu \nu$ antisymmetric tensor $\varepsilon_{\lambda \mu \nu} \varepsilon \tilde{\delta} \tilde{\varepsilon} \tilde{\phi}$, a null result is obtained so that there will be no contribution from $l=0$. In general, however, there will be contributions from $l=1,2$, and 3 , and the relaxation dynamics will be complicated. Nonetheless it is often appropriate to employ approximations in order to simplify matters, and the occurrence of symmetry within the molecule will also in general reduce the number of param- 
TABLE III. Eigenvalues (relaxation coefficients) for diffusers of different geometry and $1 \leqslant l \leqslant 3$.

\begin{tabular}{cccc}
\hline \hline & & Symmetric diffuser & Axial diffuser \\
Eigenvalue & Asymmetric diffuser & $D_{1}=D_{2}=D_{\perp}, D_{3}=D_{\|}$ & $D_{1}=D_{2}=D_{\perp}, D_{3}=\infty$ \\
\hline$E_{1}^{(1)}$ & $D_{1}+D_{3}$ & $D_{\|}+D_{\perp}$ & $\infty$ \\
$E_{0}^{(1)}$ & $D_{1}+D_{2}$ & $2 D_{\perp}$ & $2 D_{\perp}$ \\
$E_{-1}^{(1)}$ & $D_{2}+D_{3}$ & $D_{\|}+D_{\perp}$ & $\infty$ \\
$E_{2}^{(2)}$ & $6 D+2 \Delta$ & $2\left(2 D_{\|}+D_{\perp}\right)$ & $\infty$ \\
$E_{1}^{(2)}$ & $3\left(D+D_{1}\right)$ & $D_{\|}+5 D_{\perp}$ & $\infty$ \\
$E_{0}^{(2)}$ & $6 D-2 \Delta$ & $6 D_{\perp}$ & $\infty$ \\
$E_{-1}^{(2)}$ & $3\left(D+D_{2}\right)$ & $D_{\|}+5 D_{\perp}$ & $\infty$ \\
$E_{-2}^{(2)}$ & $3\left(D+D_{3}\right)$ & $2\left(2 D_{\|}+D_{\perp}\right)$ & $\infty$ \\
$E_{3}^{(3)}$ & $15 D-3 D_{2}+2 \Pi$ & $3\left(3 D_{\|}+D_{\perp}\right)$ & $\infty$ \\
$E_{2}^{(3)}$ & $15 D-3 D_{3}+2 \Xi$ & $4\left(D_{\|}+2 D_{\perp}\right)$ & $12 D_{\perp}$ \\
$E_{1}^{(3)}$ & $15 D-3 D_{2}-2 \Pi$ & $D_{\|}+11 D_{\perp}$ & $\infty$ \\
$E_{0}^{(3)}$ & $15 D-3 D_{3}-2 \Xi$ & $12 D_{\perp}$ & $\infty$ \\
$E_{-1}^{(3)}$ & $15 D-3 D_{1}-2 \Theta$ & $D_{\|}+11 D_{\perp}$ & $\infty$ \\
$E_{-2}^{(3)}$ & $12 D$ & $4\left(D_{\|}+2 D_{\perp}\right)$ & $\infty$ \\
$E_{-3}^{(3)}$ & $15 D-3 D_{1}+2 \Theta$ & $3\left(3 D_{\|}+D_{\perp}\right)$ & $\infty$ \\
\hline \hline
\end{tabular}

eters required to describe the temporal characteristics. Some important examples are considered below.

\section{A. The one-dimensional molecule}

For rodlike molecules like DEANS and DMANS (Fig. 6) a first approximation that one might consider (in common with a prevailing tradition of nonlinear optics) is that of a "one-dimensional'" molecule. Here the molecule is allowed to only have nonlinear polarizability components along the symmetry axis - which relates to the $D_{3}$ principal diffusion component. Diffusional relaxation around this axis will be infinitely fast so we may take $D_{3}$ to be infinite-symmetry also requires that relaxation perpendicular to this axis (i.e., due to tumbling motions) is isotropic, i.e., $D_{1}=D_{2}=D_{\perp}$. Thus only one component of the molecule's diffusion tensor will feature at this level of sophistication. Table III shows how such symmetry assumptions affect the relaxation rates, i.e., how the general eigenvalues $E_{\eta}^{(l)}$ vary between an asymmetrical diffusion tensor, a symmetrical diffusion tensor, and an axial (rodlike) diffusion tensor. In the one-dimensional approximation, Eqs. (80) and (81) indicate the following nonzero components:

$$
\begin{aligned}
\chi_{z z z z}^{(3)}= & \frac{\bar{\mu}_{z}^{10} \alpha_{(z z)}^{10} \Delta \beta_{z(z z)}}{105}\left(2 E_{1}+4 E_{2}-3 E_{3}\right. \\
& \left.-6 E_{4}+8 E_{5}+4 E_{6}\right), \\
\chi_{z(z z) z z z}^{(5)}= & \frac{\bar{\mu}_{z}^{10} \alpha_{(z z)}^{10} \Delta \beta_{z(z z)}}{105}\left(-E_{1}-2 E_{2}+5 E_{3}\right. \\
& \left.+10 E_{4}-4 E_{5}-2 E_{6}\right) .
\end{aligned}
$$

Thus inserting these into Eq. (79) and using Tables I, II, and III we find that the contribution from $l=2$ disappears, the ensuing expression for the second-harmonic intensity being

$$
\begin{aligned}
I_{\text {sig }}(\tau)= & \kappa\left|\bar{\mu}_{z}^{10} \alpha_{(z z)}^{10} \Delta \beta_{z(z z)} / 525\right|^{2} \mid 7\left(E_{1}+2 E_{2}+4 E_{5}+2 E_{6}\right) \\
& \times e^{-2 D_{\perp} \tau}+2\left(-E_{1}-2 E_{2}+5 E_{3}+10 E_{4}\right. \\
& \left.-4 E_{5}-2 E_{6}\right)\left.e^{-12 D_{\perp} \tau}\right|^{2} .
\end{aligned}
$$

In this approximation, we have contributions only from odd $l$ values and we expect to find an SHG amplitude that relaxes with a biexponential decay, in agreement with classical theory treatments and also our experimental observations in DMANS $[5,6]$. The relaxation rates are predicted to be in the ratio of $6: 1$. To observe this feature it is useful to consider two specific polarization combinations [5]. If all beams have linear vertical polarization in the laboratory $X$ direction, the signal intensity $I_{\text {sig }}(\tau)$ takes the form

$$
I_{\mathrm{sig}}^{\|}(\tau)=\kappa\left|\bar{\mu}_{z}^{10} \alpha_{(z z)}^{10} \Delta \beta_{z(z z)} / 175\right|^{2}\left|21 e^{-2 D_{\perp} \tau}+4 e^{-12 D_{\perp} \tau}\right|^{2},
$$

whereas if beam $l$ is linearly polarized at $45^{\circ}$ to the vertical and the horizontal component of the signal is measured while keeping the other two beams unchanged, the intensity $I_{\text {sig }}^{\perp}(\tau)$ is given by

$$
\begin{aligned}
I_{\text {sig }}^{\perp}(\tau)= & \kappa\left|\bar{\mu}_{z}^{10} \alpha_{(z z)}^{10} \Delta \beta_{z(z z)} / 175\right|^{2} \\
& \times \cos ^{2} \delta\left|7 e^{-2 D_{\perp} \tau}-2 e^{-12 D_{\perp} \tau}\right|^{2} .
\end{aligned}
$$

The ratio of the amplitudes associated with these signals is hence

$$
\begin{aligned}
r(\tau) & =\sqrt{I_{\mathrm{sig}}^{\|}(\tau) / I_{\mathrm{sig}}^{\perp}(\tau)}=\left|\frac{21+4 e^{-10 D_{\perp} \tau}}{\left(7-2 e^{-10 D_{\perp} \tau}\right) \cos \delta}\right| \\
& \approx\left|\frac{21+4 e^{-10 D_{\perp} \tau}}{7-2 e^{-10 D_{\perp} \tau}}\right|
\end{aligned}
$$


for small angles $\delta$. The agreement between Eqs. (85) and (86) and experiment is not good in the case of polar solvents [5] - these equations predict a biexponential SHG amplitude with decay rates in the ratio $6: 1$, as against experimental observations in THF where biexponential decay has been recorded with relative decay rates nearer to $40: 1$ [5]. Evidently, there is a fast relaxation channel not manifest in the simple one-dimensional model. The temporal variation of $r$, however, has been reported previously for DMANS and this agrees well with Eq. (87). This observation, along with measurements in nonpolar solvents, has been used as evidence for a time- and solvent polarity-dependent hyperpolarizability [5,6], $\Delta \beta_{z(z z)}=\Delta \beta_{z(z z)}(t)$. An alternative explanation might be that the one-dimensional model may break down for a real molecule like DEANS. Next we shall look at the consequences of relaxing such constraints.

\section{B. Linear molecules}

The one-dimensional model is clearly very primitiveeven a linear polar molecule in an optical field possesses, in addition to its transition dipole moment $\mu=\mu_{z}^{10}$, three nonvanishing polarizability components $\alpha_{\|}=\alpha_{(z z)}^{10}$ and $\alpha_{\perp}$ $=\alpha_{(x x)}^{10}=\alpha_{(y y)}^{10}$, and seven nonvanishing hyperpolarizability components [27], $\Delta \beta_{\|}=\Delta \beta_{z(z z)}, \Delta \beta_{\perp}=\Delta \beta_{z(x x)}=\Delta \beta_{z(y y)}$, $\Delta \beta_{x}=\Delta \beta_{x(z x)}=\Delta \beta_{x(x z)}=\Delta \beta_{y(z y)}=\Delta \beta_{y(y z)}$. Introducing these components in Eq. (79) produces the following expression for the SHG intensity:

$$
I_{\text {sig }}(\tau)=\kappa\left|\mu_{\|} / 525\right|^{2}\left|7 K_{1} e^{-2 D_{\perp} \tau}+2 K_{2} e^{-12 D_{\perp} \tau}\right|^{2},
$$

where the polarizability and field-dependent constants are given by

$$
K_{1}=\left[\begin{array}{c}
\alpha_{\|} \Delta \beta_{\|} \\
\alpha_{\perp} \Delta \beta_{\|} \\
\alpha_{\|} \Delta \beta_{\perp} \\
\alpha_{\perp} \Delta \beta_{\perp} \\
\alpha_{\|} \Delta \beta_{x} \\
\alpha_{\perp} \Delta \beta_{x}
\end{array}\right]^{T}\left[\begin{array}{cccc}
1 & 2 & 4 & 2 \\
4 & -2 & -4 & 8 \\
4 & 8 & -4 & -2 \\
16 & -8 & 4 & -8 \\
-2 & -4 & 12 & 6 \\
-8 & 4 & -12 & 24
\end{array}\right]\left[\begin{array}{c}
E_{1} \\
E_{2} \\
E_{5} \\
E_{6}
\end{array}\right],
$$

$$
\begin{aligned}
K_{2}= & \left(\alpha_{\|}-\alpha_{\perp}\right)\left(\Delta \beta_{\|}-\Delta \beta_{\perp}-2 \Delta \beta_{x}\right) \\
& \times\left(-E_{1}-2 E_{2}+5 E_{3}+10 E_{4}-4 E_{5}-2 E_{6}\right) .
\end{aligned}
$$

Recomputing the ratio given in Eq. (87), assuming $\cos ^{2} \delta$ $\approx 1$, we obtain

$$
r(\tau)=\sqrt{I_{\mathrm{sig}}^{\|}(\tau) / I_{\mathrm{sig}}^{\perp}(\tau)}=\left|\frac{R_{1}+4 e^{-10 D_{\perp} \tau}}{R_{2}-2 e^{-10 D_{\perp} \tau}}\right|
$$

where

$$
\begin{array}{r}
R_{1}=\frac{7\left(9 \alpha_{\|} \Delta \beta_{\|}+6 \alpha_{\perp} \Delta \beta_{\|}+6 \alpha_{\|} \Delta \beta_{\perp}+4 \alpha_{\perp} \Delta \beta_{\perp}+12 \alpha_{\|} \Delta \beta_{x}+8 \alpha_{\perp} \Delta \beta_{x}\right)}{3\left(\alpha_{\|}-\alpha_{\perp}\right)\left(\Delta \beta_{\|}-\Delta \beta_{\perp}-2 \Delta \beta_{x}\right)}, \\
R_{2}=\frac{7\left(6 \alpha_{\|} \Delta \beta_{\|}+4 \alpha_{\perp} \Delta \beta_{\|}-6 \alpha_{\|} \Delta \beta_{\perp}-4 \alpha_{\perp} \Delta \beta_{\perp}+18 \alpha_{\|} \Delta \beta_{x}+12 \alpha_{\perp} \Delta \beta_{x}\right)}{6\left(\alpha_{\|}-\alpha_{\perp}\right)\left(\Delta \beta_{\|}-\Delta \beta_{\perp}-2 \Delta \beta_{x}\right)} .
\end{array}
$$

For the one-dimensional model only $\alpha_{\|}$and $\Delta \beta_{\|}$are nonzero and these reduce to $R_{1}=21$ and $R_{2}=7$ as required. Deviations from these values are to be expected for any real molecule undergoing rotational reorientation. One may therefore ask how sensitive these quantities should be to the shape of the relaxing molecule.

For an initial exploration of this complex issue let us examine a model wherein each nonaxial polarizability component bears the same ratio to the axial component-thus we suppose that $\alpha_{\perp}=q \alpha_{\|}$and $\Delta \beta_{\perp}=\Delta \beta_{x}=q \Delta \beta_{\|}$, where $q$ is a parameter reflecting the divergence from the onedimensional axial model $(q=0)$. In terms of this parameter we find that the signal intensity takes the form

$$
I_{\text {sig }}(\tau)=\kappa\left|\mu_{\|} \alpha_{\|} \Delta \beta_{\|} / 525\right|^{2}\left|7 K_{1}^{\prime} e^{-2 D_{\perp} \tau}+2 K_{2}^{\prime} e^{-12 D_{\perp} \tau}\right|^{2},
$$

where

$$
K_{1}^{\prime}=\left[\begin{array}{c}
1 \\
q \\
q^{2}
\end{array}\right]^{T}\left[\begin{array}{cccc}
1 & 2 & 4 & 1 \\
6 & 2 & 4 & 12 \\
8 & -4 & -8 & 16
\end{array}\right]\left[\begin{array}{c}
E_{1} \\
E_{2} \\
E_{5} \\
E_{6}
\end{array}\right]
$$

and

$$
\begin{aligned}
K_{2}^{\prime}= & (1-q)(1-3 q)\left(-E_{1}-2 E_{2}+5 E_{3}\right. \\
& \left.+10 E_{4}-4 E_{5}-2 E_{6}\right) .
\end{aligned}
$$

Using these expressions we find that the intensities $I_{\text {sig }}^{\|}(\tau)$ and $I_{\text {sig }}^{\perp}(\tau)$, described by Eqs. (85) and (86), respectively, take the form

$$
\begin{aligned}
I_{\mathrm{sig}}^{\|}(\tau)= & \kappa\left|\mu_{\|} \alpha_{\|} \Delta \beta_{\|} / 175\right|^{2} \mid 7(1+2 q)(3+2 q) e^{-2 D_{\perp} \tau} \\
& +\left.4(1-q)(1-3 q) e^{-12 D_{\perp} \tau}\right|^{2}
\end{aligned}
$$




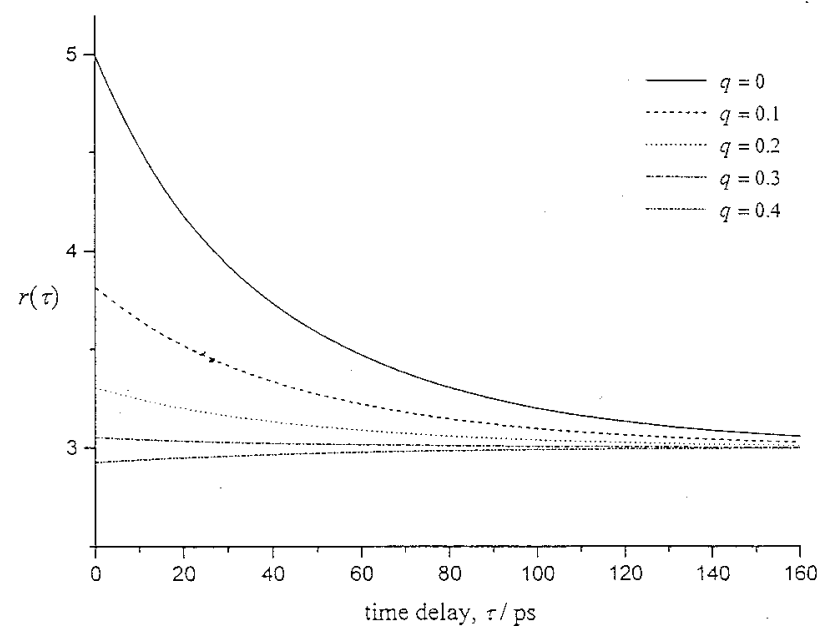

FIG. 4. Variation of the ratio $r(\tau)$ [Eq. (99)] for different $q$ values. A diffusion coefficient $D_{\perp}=2.0 \times 10^{-3} \mathrm{ps}^{-1}$ has been assumed as appropriate for a solution of DEANS in mesitylene.

$$
\begin{aligned}
I_{\text {sig }}^{\perp}(\tau)= & \kappa\left|\mu_{\|} \alpha_{\|} \Delta \beta_{\|} / 525\right|^{2} \mid 7(1+2 q)(3+2 q) e^{-2 D_{\perp} \tau} \\
& -\left.6(1-q)(1-3 q) e^{-12 D_{\perp} \tau}\right|^{2} \cos ^{2} \delta
\end{aligned}
$$

and so, again assuming $\cos ^{2} \delta \approx 1$, we have

$$
r(\tau)=\left|\frac{21(1+2 q)(3+2 q)+12(1-q)(1-3 q) e^{-10 D_{\perp} \tau}}{7(1+2 q)(3+2 q)-6(1-q)(1-3 q) e^{-10 D_{\perp} \tau}}\right| .
$$

The variation of this ratio is shown in Fig. 4 for a range of $q$ values. We see that even a small deviation from the onedimensional model will lead to temporal characteristics significantly different from those predicted by the onedimensional model. Figure 5 shows the results of a fit to Eqs. (97) and (98) of data collected from a solution of DEANS in mesitylene [6]. The data were not collected under the stringent conditions required to generate a meaningful ratio $r(\tau)$, but the fit to the individual amplitudes is seen to be good and yields a consistent value for the parameter $q=0.017$ \pm 0.007 . Although the error here is large, the value of $q$ is significantly different from zero. Thus, although the analysis implies an axial $\Delta \beta_{z z z}$ component $42-100$ times larger than the other nonzero components, it is apparent that the small off-axial components do significantly affect the dynamics. It therefore has to be assumed that a similar conclusion would be drawn in any more detailed representation.

\section{A nonlinear model}

The nonlinear model that we shall consider, illustrated in Fig. 6, allows for distinct diffusion coefficients to be associated with each of the three axes. The molecule is treated as if planar and, for the evaluation of polarizabilities, of $C_{2 v}$ symmetry. The components of the polarizability tensors that are nonzero are thus [28]:

$$
\mu_{\|}=\mu_{z z}, \quad \alpha_{\|}=\alpha_{(z z)}^{10}, \quad \alpha_{\perp}^{(1)}=\alpha_{(x x)}^{10}, \quad \alpha_{\perp}^{(2)}=\alpha_{(y y)}^{10},
$$

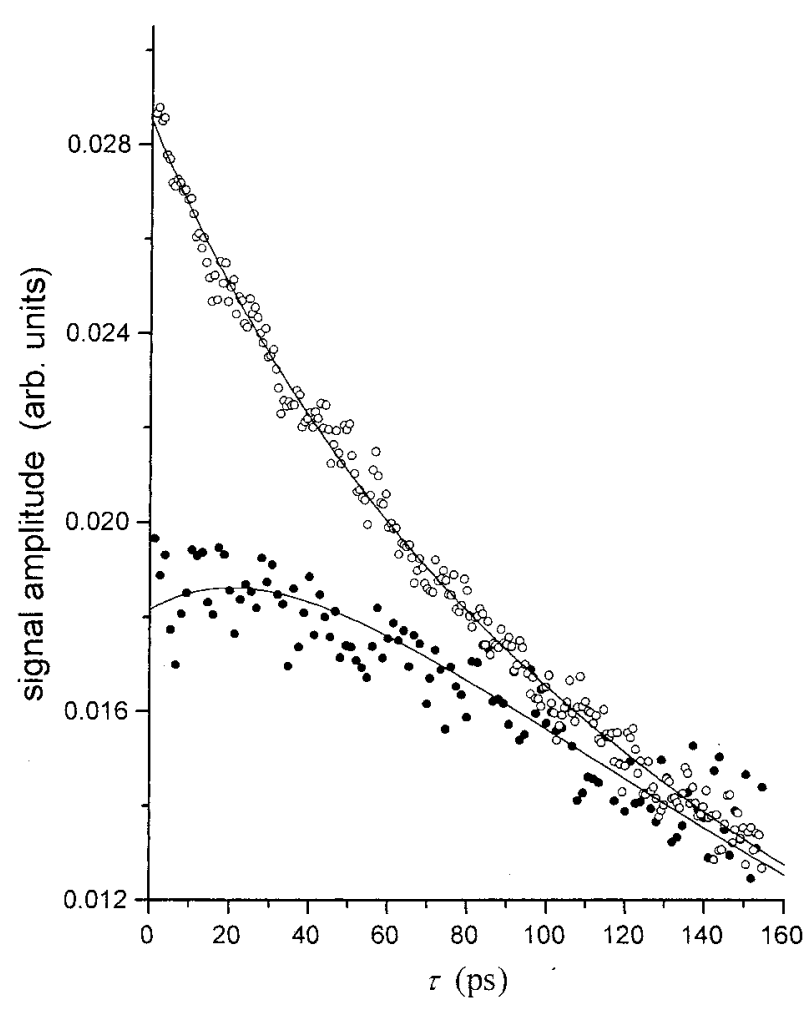

FIG. 5. The time dependence of the SHG amplitudes for two different polarization arrangements for a solution of DEANS in mesitylene: $(\bigcirc)$ are the measured data for all vertical polarizations and () for beams 1 and 2 vertical, beam 3 at $45^{\circ}$, and detection of the horizontal component of beam 4 . The solid lines are fits to Eqs. (97) and (98) using $D_{\perp}=2.0 \times 10^{-3} \mathrm{ps}^{-1}$, yielding $q=0.017$ \pm 0.007 .

$$
\begin{gathered}
\Delta \beta_{\|}=\Delta \beta_{z(z z)}, \quad \Delta \beta_{\perp}^{(1)}=\Delta \beta_{z(x x)}=\Delta \beta_{x(x z)}=\Delta \beta_{x(z x)}, \\
\Delta \beta_{\perp}^{(2)}=\Delta \beta_{z(y y)}=\Delta \beta_{y(y z)}=\Delta \beta_{y(z y)} .
\end{gathered}
$$

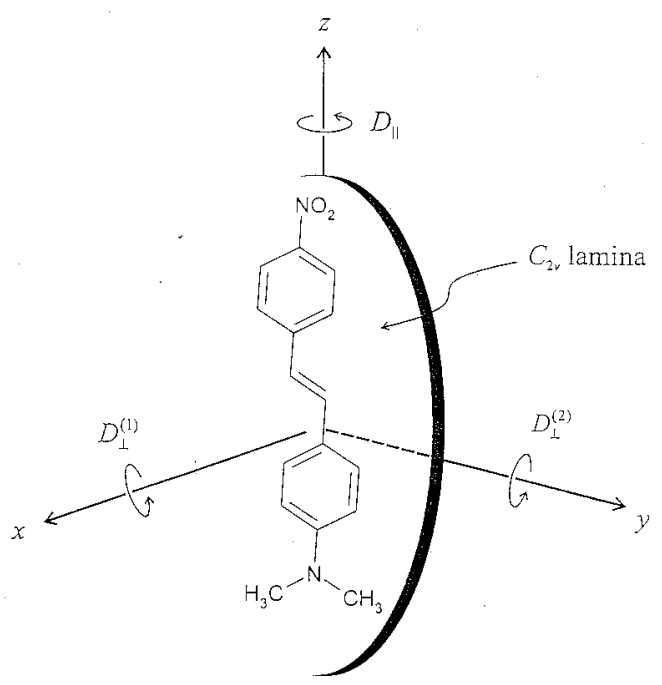

FIG. 6. A simple nonlinear geometry used to model the dynamics of the DMANS molecule. 
For this model the second-harmonic amplitude decays as a triexponential:

$$
\begin{aligned}
I_{\text {sig }}(\tau)= & \kappa \mid f_{\text {fast }} e^{-\left(15 D-3 D_{\|}+2 \Xi\right) \tau}+f_{\text {int }} e^{-\left(15 D-3 D_{\|}-2 \Xi\right) \tau} \\
& +\left.f_{\text {slow }} e^{-\left(D_{\perp}^{(1)}+D_{\perp}^{(2)}\right)}\right|^{2}
\end{aligned}
$$

where the terminology of Table I has been used so that

$$
D=\left(D_{\perp}^{(1)}+D_{\perp}^{(2)}+D_{\|}\right) / 3
$$

and

$$
\Xi^{2}=4 D_{\perp}^{(1)^{2}}+4 D_{\perp}^{(2)^{2}}+D_{\|}^{2}-7 D_{\perp}^{(1)} D_{\perp}^{(2)}-D_{\perp}^{(1)} D_{\|}-D_{\perp}^{(2)} D_{\|} .
$$

Here $D_{\perp}^{(1)}$ and $D_{\perp}^{(2)}$ represent diffusion coefficients corresponding to tumbling motions and $D_{\|}$is associated with ro- tation about the molecular spine. In Eq. (100) the preexponential coefficients are labeled in such a manner as to indicate the relative rates of the decay terms. Indeed, as the diffusion coefficients approach the linear case, we have from Table III

$$
\begin{gathered}
15 D-3 D_{\|}+2 \Xi \underset{\text { linear }}{\rightarrow \infty}, \quad 15 D-3 D_{\|}-2 \Xi \underset{\text { linear }}{\rightarrow} 12 D_{\perp}, \\
D_{\perp}^{(1)}+D_{\perp}^{(2)} \underset{\text { linear }}{\rightarrow} 2 D_{\perp} .
\end{gathered}
$$

However this more sophisticated model is capable of accounting for the occurrence of an additional decay component in the six-wave mixing data that are expected for molecules of arbitrary shape.

The preexponential coefficients in Eq. (100) are given by

$$
\begin{gathered}
f_{\text {slow }}=\frac{\mu_{\|}\left(\Delta \beta_{\perp}^{(1)}+\Delta \beta_{\perp}^{(2)}+\Delta \beta_{\|}\right)}{75}\left\{\alpha_{\|}\left(E_{1}+2 E_{2}+4 E_{5}+2 E_{6}\right)+\left(\alpha_{\perp}^{(1)}+\alpha_{\perp}^{(2)}\right)\left(2 E_{1}-E_{2}-2 E_{5}+4 E_{6}\right)\right\}, \\
f_{\text {int }}=\frac{\mu_{\|}\left(-E_{1}-2 E_{2}+5 E_{3}+10 E_{4}-4 E_{5}-2 E_{6}\right)}{1050\left(15 s^{2}+1\right)}\left\{3 \alpha_{\perp}^{(1)} \Delta \beta_{\perp}^{(1)}(s+1)^{2}-3 \alpha_{\perp}^{(1)} \Delta \beta_{\perp}^{(2)}\left(s^{2}-1\right)-2 \alpha_{\perp}^{(1)} \Delta \beta_{\|}(s+1)\right. \\
\left.-3 \alpha_{\perp}^{(2)} \Delta \beta_{\perp}^{(1)}\left(s^{2}-1\right)+3 \alpha_{\perp}^{2} \Delta \beta_{\perp}^{(2)}(s-1)^{2}+2 \alpha_{\perp}^{(2)} \Delta \beta_{\|}(s-1)-6 \alpha_{\|} \Delta \beta_{\perp}^{(1)}(s+1)+6 \alpha_{\|} \Delta \beta_{\perp}^{(2)}(s-1)+4 \alpha_{\|} \Delta \beta_{\|}\right\} \\
f_{\text {fast }}=\frac{\mu_{\|}\left(-E_{1}-2 E_{2}+5 E_{3}+10 E_{4}-4 E_{5}-2 E_{6}\right)}{210\left(15 s^{2}+1\right)}\left\{\alpha_{\perp}^{(1)} \Delta \beta_{\perp}^{(1)}(3 s-1)^{2}+\alpha_{\perp}^{(1)} \Delta \beta_{\perp}^{(2)}\left(9 s^{2}-1\right)-2 \alpha_{\perp}^{(1)} \Delta \beta_{\|} s(3 s-1)+\alpha_{\perp}^{(2)} \Delta \beta_{\perp}^{(1)}\right. \\
\left.\times\left(9 s^{2}-1\right)+\alpha_{\perp}^{(2)} \Delta \beta_{\perp}^{(2)}(3 s+1)^{2}-2 \alpha_{\perp}^{2} \Delta \beta_{\|} s(3 s+1)-6 \alpha_{\|} \Delta \beta_{\perp}^{(1)} s(3 s-1)-6 \alpha_{\|} \Delta \beta_{\perp}^{(2)} s(3 s+1)+12 \alpha_{\|} \Delta \beta_{\|} s^{2}\right\},
\end{gathered}
$$

where $s$ is a diffusion parameter defined as

$$
s=\frac{\left(D_{\perp}^{(1)}-D_{\perp}^{(2)}\right)}{2 \Xi-D_{\perp}^{(1)}-D_{\perp}^{(2)}+2 D_{\|}} .
$$

For symmetric diffusers $D_{\perp}^{(1)}=D_{\perp}^{(2)}$ and $s$ disappears. Table III indicates that for such molecules the fast decay coefficient will be $4\left(D_{\|}+2 D_{\perp}\right)$. Equation (105) also reduces to

$$
\begin{aligned}
f_{\text {fast }}= & \frac{\mu_{\|}\left(-E_{1}-2 E_{2}+5 E_{3}+10 E_{4}-4 E_{5}-2 E_{6}\right)}{210} \\
& \times\left(\alpha_{\perp}^{(1)}-\alpha_{\perp}^{(2)}\right)\left(\Delta \beta_{\perp}^{(1)}-\Delta \beta_{\perp}^{(2)}\right) .
\end{aligned}
$$

The fast term thus disappears in the one-dimensional and linear models, but a fast component in the orientational relaxation may well arise for more complex molecular shapes.

\section{SUMMARY}

A quantum-electrodynamical treatment of an ultrafast time-resolved six-wave mixing experiment has been presented. Consideration of the interference of two excitation pathways leads naturally into a description quite analogous to the classical transient grating picture of time-resolved experiments. It is, however, straightforward to incorporate the polarization dependence of the signal into the QED treatment [16].

To account for the time-resolved data we have also incorporated into the analysis the population dynamics and the dynamics of diffusional reorientation. The reorientational dynamics are complex but several limiting cases have been treated. For a one-dimensional molecule the decay of the six-wave signal is predicted to follow biexponential relaxation with a 6:1 ratio of relaxation times. This is as observed experimentally. However, it has also been shown that by allowing for the existence of nonaxial components of the polarizability, somewhat better agreement with experiment is obtained. It has furthermore been demonstrated that for a 
molecule characterized by three different diffusion coefficients the dynamics may exhibit a triexponential form. Thus the theory presented is capable of correctly accounting for the rotational dynamics of molecules of arbitrary shape, observed through six-wave mixing. Future applications will include a more detailed study of the polarization dependence of the reorientational dynamics. In addition we plan to incorporate into the theory the possibility of nondiffusive (libra- tional) orientational motion, as well as the possibility of a time-dependent hyperpolarizability [5].

\section{ACKNOWLEDGMENT}

This work was funded by a research grant from the Science and Engineering Research Council.
[1] K. Tominaga and K. Yoshihara, Phys. Rev. Lett. 74, 3061 (1995).

[2] K. Tominaga and K. Yoshihara, J. Chem. Phys. 104, 4419 (1996).

[3] T. Steffen and K. Duppen, Phys. Rev. Lett. 76, 1224 (1996).

[4] T. Steffen and K. Duppen, J. Chem. Phys. 106, 3854 (1997).

[5] S. Lin, I. D. Hands, D. L. Andrews, and S. R. Meech, Chem. Phys. Lett. 285, 321 (1998).

[6] S. Lin, I. D. Hands, D. L. Andrews, and S. R. Meech, J. Phys. Chem. A 103, 3830 (1999).

[7] C. Fiorini, F. Charra, and J.-M. Nunzi, J. Opt. Soc. Am. B 11, 2347 (1994).

[8] C. Fiorini, F. Charra, J.-M. Nunzi, and P. Raimond, J. Opt. Soc. Am. B 14, 1984 (1997).

[9] A. Tokmakoff, M. J. Lang, X. J. Jordanides, and G. R. Fleming, Chem. Phys. 233, 231 (1998).

[10] M. Yang, J. Y. Kim, Y. Jung, and M. H. Cho, J. Chem. Phys. 108, 4013 (1998).

[11] W. M. Zhang, V. Chernyak, and S. Mukamel, J. Chem. Phys. 110, 5011 (1999).

[12] T. Steffen, Ph.D. thesis, University of Groningen, 1998 (unpublished).

[13] D. A. Blank, L. J. Kaufman, and G. R. Fleming, J. Chem. Phys. 111, 3105 (1999).

[14] J. C. Kirkwood, A. C. Albrecht, and D. J. Ulness, J. Chem. Phys. 111, 253 (1999).
[15] J. C. Kirkwood, A. C. Albrecht, D. J. Ulness, and M. J. Stimson, J. Chem. Phys. 111, 272 (1999).

[16] I. D. Hands, S. Lin, S. R. Meech, and D. L. Andrews, J. Chem. Phys. 109, 10580 (1998).

[17] R. L. Murry and J. T. Fourkas, J. Chem. Phys. 107, 9726 (1997).

[18] L. D. Favro, Phys. Rev. 119, 53 (1960).

[19] S. Lin, I. D. Hands, D. L. Andrews, and S. R. Meech, Opt. Commun. 174, 285 (2000).

[20] D. L. Andrews, S. Naguleswaran, and G. E. Stedman, Phys. Rev. A 57, 4925 (1998).

[21] G. E. Stedman, S. Naguleswaran, D. L. Andrews, and L. C. Dávila Romero (unpublished).

[22] P. Allcock and D. L. Andrews, J. Phys. B 30, 3731 (1997).

[23] D. P. Craig and T. Thirunamachandran, Molecular Quantum Electrodynamics (Dover, New York, 1998).

[24] W. T. Huntress, Jr., in Advances in Magnetic Resonance, edited by J. S. Waugh (Academic, New York, 1970), Vol. 4, p. 1.

[25] I. S. Gradshteyn and I. M. Ryzhik, Table of Integrals Series and Products (Academic, London, 1983), p. 948.

[26] S. Kielich, Bull. Acad. Soc. Amis Sci. Lett. Poznan Ser. B 21, 47 (1968).

[27] A. D. Buckingham, in Nonlinear Behaviour of Molecules, Atoms and Ions in Electric Magnetic or Electromagnetic Fields, edited by L. Néel (Elsevier, Amsterdam, 1979), p. 217.

[28] L. D. Barron, Molecular Light Scattering and Optical Activity (Cambridge University Press, Cambridge, 1982), p. 205. 